\title{
STOKES COMPLEXES AND THE CONSTRUCTION OF STABLE FINITE ELEMENTS WITH POINTWISE MASS CONSERVATION *
}

\author{
RICHARD S. FALK ${ }^{\dagger}$ AND MICHAEL NEILAN ${ }^{\ddagger}$
}

\begin{abstract}
Two families of conforming finite elements for the two-dimensional Stokes problem are developed, guided by two discrete smoothed de Rham complexes, which we coin "Stokes complexes." We show that the finite element pairs are inf-sup stable and also provide pointwise mass conservation on very general triangular meshes.
\end{abstract}

Key words. finite elements, Stokes, conforming, divergence-free

AMS subject classifications. $76 \mathrm{M} 10,65 \mathrm{~N} 30,65 \mathrm{~N} 12$

1. Introduction. In this paper we develop two families of stable finite element methods for the stationary Stokes equations with no-slip boundary conditions:

$$
\begin{array}{rlrl}
-\nu \Delta \boldsymbol{u}+\nabla p & =\boldsymbol{f} & & \text { in } \Omega, \\
\operatorname{div} \boldsymbol{u}=0 & & \text { in } \Omega, \\
\boldsymbol{u} & =0 & & \text { on } \partial \Omega .
\end{array}
$$

Here $\Omega \subset \mathbb{R}^{2}$ is a simply connected polyhedral domain, $\boldsymbol{u}$ and $p$ denote the velocity and the pressure of the fluid, respectively, $\nu>0$ is the kinematic viscosity (assumed to be constant for simplicity), and $\boldsymbol{f} \in \boldsymbol{L}^{2}(\Omega)$ is the external body force. A detailed description of the notation used throughout the paper is given in the following section.

Our specific goal is to construct finite element pairs $\boldsymbol{X}_{h, 0} \times Y_{h, 0}$ consisting of piecewise polynomials with respect to a simplicial triangulation that

(A1) are conforming, i.e., $\boldsymbol{X}_{h, 0} \subset \boldsymbol{H}_{0}^{1}(\Omega)$ and $Y_{h, 0} \subset \stackrel{\circ}{L}^{2}(\Omega)$;

(A2) are stable; that is the Ladyzenskaja-Babuska-Brezzi (LBB) condition

$$
C\|q\|_{L^{2}(\Omega)} \leq \sup _{\boldsymbol{v} \in \boldsymbol{X}_{h, 0} \backslash\{0\}} \frac{\int_{\Omega}(\operatorname{div} \boldsymbol{v}) q d x}{\|\boldsymbol{v}\|_{H^{1}(\Omega)}} \quad \forall q \in Y_{h, 0}
$$

is satisfied for a constant $C>0$ independent of $h$;

(A3) produce divergence-free pointwise velocity approximations for the Stokes problem.

Furthermore, we construct finite element spaces that satisfy these properties on very general triangulations.

Conditions (A2)-(A3) address two types of numerical instabilities of the Stokes problem (1.1). Condition (A2) implies that the divergence operator div : $\boldsymbol{X}_{h, 0} \rightarrow Y_{h, 0}$ is surjective onto the pressure space $Y_{h, 0}$. It eliminates the so-called spurious pressure modes and is a necessary and sufficient condition for the well-posedness of the discrete problem $[8,5]$. Condition (A3), on the other hand, suggests the reverse relation $Y_{h, 0} \subset \operatorname{div} \boldsymbol{X}_{h, 0}$, and addresses the numerical instability due to poor mass conservation. The enforcement of this condition (or the lack-thereof) has been shown to have dramatic consequences, even at moderate Reynolds numbers [17, 18, 11, 21]. More precisely, it is

${ }^{*}$ This work was supported by the National Science Foundation through grants DMS-0910540 (Falk) and DMS1238711 (Neilan).

$\dagger$ Department of Mathematics, Rutgers University, Piscataway, NJ 08854 (falk@math.rutgers.edu).

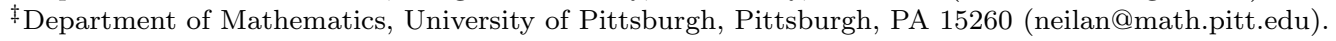


well-known that if (A1)-(A2) is satisfied, but mass conservation is only enforced weakly, then the error of the velocity approximation satisfies

$$
\left\|\boldsymbol{u}-\boldsymbol{u}_{h}\right\|_{H^{1}(\Omega)} \leq C\left(\inf _{\boldsymbol{v} \in \boldsymbol{X}_{h, 0}}\|\boldsymbol{u}-\boldsymbol{v}\|_{H^{1}(\Omega)}+\nu^{-1} \inf _{q \in Y_{h, 0}}\|p-q\|_{L^{2}(\Omega)}\right) .
$$

If the viscosity $\nu$ is large and/or the pressure gradient is small, then the second term in the righthand side becomes negligible. In the other cases, the error scales like $\mathcal{O}\left(\nu^{-1}\right)$.

Over the past 40 years, many finite element methods have been developed based upon the velocity-pressure formulation of the Stokes problem on triangular meshes (cf. [15, 5, 8]). However, the majority of these methods only satisfy conditions (A1)-(A2); the third condition is only imposed weakly. Methods that fall into this category include the well-known Taylor-Hood elements [5], the MINI element [2], the Bernardi-Raugel elements [4], the Crouzeix-Raviert elements [8, VI Example 3.6], and the $\mathcal{P}_{2}-\mathcal{P}_{0}$ finite element pair [5]. On the other hand, it was shown by Scott and Vogelius $[22,25]$ that the $\mathcal{P}_{k}-\mathcal{P}_{k-1}$ pair (with $\mathcal{P}_{k}$ globally continuous and $\mathcal{P}_{k-1}$ discontinuous) satisfy (A1)-(A3) provided (i) the polynomial degree $k$ is greater or equal to four, (ii) the triangulation is quasi-uniform and (iii) the triangulation does not contain any singular vertices (i.e., vertices that fall on exactly two straight lines). It was later shown that the spaces $\mathcal{P}_{k}-\mathcal{P}_{k-1}$ satisfy these conditions for smaller values of $k$ on very specific types of uniform triangulations [3]. Very recently, Guzmán and the second author constructed a family of finite elements that satisfy all three conditions on arbitrary shape-regular triangulations. These properties are achieved by adding divergence-free rational functions to $\boldsymbol{H}(\operatorname{div} ; \Omega)$-conforming finite element spaces. As far as we are aware, the Scott-Vogelius elements and the Guzmán-Neilan elements are the only class of elements that satisfy (A1)-(A3). We remark that discontinuous Galerkin methods [9, 10] and isogeometric methods $[13,14]$ have been constructed for the Stokes problem that produce exactly divergence-free velocity approximations.

Similar to the Scott-Vogelius elements, we also consider globally continuous piecewise polynomials of degree $k$ to approximate the velocity and piecewise polynomials of degree $k-1$ to approximate the pressure. Also similar is the requirement $k \geq 4$. The key difference of our approach is that we enforce higher regularity at the vertices of the triangulation, namely, the velocity and pressure spaces are, respectively, $C^{1}$ and $C^{0}$ at the vertices. Due to this enhanced regularity, we are able to show that the elements are stable on a general class of triangulations. The only assumption we make is that the triangulation does not have any singular corner vertices. Equivalently, we require that each triangle of the triangulation has at most one boundary edge. We note that a similar assumption is required to carry out the analysis of the Taylor-Hood elements (cf. [20, 6]). Besides the standard shape regularity assumption, this is the only assumption we make on the triangulation; quasi-uniform meshes are not required to carry out our analysis. The elements we derive have the disadvantage that they require vertex degrees of freedom for the derivatives of the velocity functions, and are therefore not affine equivalent. On the other hand, they have significantly fewer global degrees of freedom than the Scott-Vogelius elements.

The construction of our elements are closely related to two different smoothed de Rham complexes ("Stokes complexes"). The first complex, originally introduced by Tai and Winther [24] to develop non-conforming methods for the Brinkman problem, is given by

$$
\mathbb{R} \stackrel{\subset}{\longrightarrow} H^{2}(\Omega) \stackrel{\text { curl }}{\longrightarrow} H^{1}(\Omega) \stackrel{\text { div }}{\longrightarrow} L^{2}(\Omega) \longrightarrow 0 .
$$

The statement that (1.3) is a complex just means that the composition of two consecutive mappings is zero. The complex is exact provided the domain $\Omega$ is simply connected [15, 24]; that is, the range 
of each map is the null space of the succeeding map. In particular, the exactness of the sequence implies that (i) for every $q \in L^{2}(\Omega)$ there exists $\boldsymbol{v} \in \boldsymbol{H}^{1}(\Omega)$ such that $\operatorname{div} \boldsymbol{v}=q$ and (ii) if $\boldsymbol{v} \in \boldsymbol{H}^{1}(\Omega)$ and $\operatorname{div} \boldsymbol{v}=0$, then $\boldsymbol{v}=\operatorname{curl} z$ for some $z \in H^{2}(\Omega)$. In addition, it is easy to see that

$$
\mathbb{R} \stackrel{\subset}{\longrightarrow} H^{2}(\Omega) \stackrel{\text { curl }}{\longrightarrow} H^{1}(\operatorname{div} ; \Omega) \stackrel{\text { div }}{\longrightarrow} H^{1}(\Omega) \longrightarrow 0,
$$

is also a complex. Here, $\boldsymbol{H}^{1}(\operatorname{div} ; \Omega)$ consists of all vector-valued $\boldsymbol{H}^{1}(\Omega)$ functions with divergence in $H^{1}(\Omega)$. The exactness of the sequence (1.3) implies the exactness of (1.4). Indeed, for $q \in$ $H^{1}(\Omega) \subset L^{2}(\Omega)$ there exists $\boldsymbol{v} \in \boldsymbol{H}^{1}(\Omega)$ with $\operatorname{div} \boldsymbol{v}=q$. Then trivially $\boldsymbol{v} \in \boldsymbol{H}^{1}(\operatorname{div} ; \Omega)$. On the other hand, if $\boldsymbol{v} \in \boldsymbol{H}^{1}(\Omega)$ is a solenoidal function, then obviously $\boldsymbol{v} \in \boldsymbol{H}^{1}(\operatorname{div} ; \Omega)$. It then follows from (1.3) that $\boldsymbol{v}=\operatorname{curl} z$ for some $z \in H^{2}(\Omega)$.

A valuable tool to design stable finite element discretizations for the Stokes problem is to find analogous discrete sub-complexes of the Stokes complex consisting of finite element spaces; i.e.,

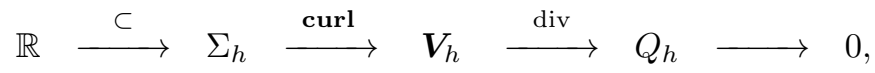

and

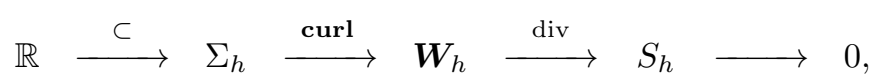

with $\Sigma_{h} \subset H^{2}(\Omega), \boldsymbol{V}_{h} \subset \boldsymbol{H}^{1}(\Omega), Q_{h} \subset L^{2}(\Omega), \boldsymbol{W}_{h} \subset \boldsymbol{H}^{1}(\operatorname{div} ; \Omega)$ and $S_{h} \subset H^{1}(\Omega)$. We point out that the exactness of the discrete complexes imply condition (A3) above. A natural starting point is to take $\Sigma_{h}$ to be the generalized Argryis space of degree $k+1$ with $k \geq 4$ (also known as the TUBA family) $[12,7,1]$. These spaces consist of globally $C^{1}$ piecewise polynomials of degree $k+1(k \geq 4)$ that are $C^{2}$ at the vertices of the triangulation.

The organization of the paper is as follows. After laying out the notation and stating some preliminary results in Section 2, we introduce the family of $\boldsymbol{H}^{1}(\Omega) \times L^{2}(\Omega)$ conforming finite element pairs in Section 3. Here we state the spaces and their corresponding degrees of freedom, and prove the LBB condition (1.2). In Section 4 we modify these spaces to construct a family of $\boldsymbol{H}^{1}(\operatorname{div} ; \Omega) \times H^{1}(\Omega)$-conforming Stokes elements and prove analogous results. Section 5 is devoted to the convergence analysis of the Stokes problem. In Section 6 we briefly describe a variant of the lowest order elements with less degrees of freedom. We provide some numerical experiments in Section 7 which back up the theoretical results, and end the paper with an overview and some extensions in Section 8.

2. Preliminaries. For an open set $S \subset \Omega$ and non-negative integer $m$, we denote by $H^{m}(S)$ the Hilbert space consisting of all $L^{2}(S)$ functions whose distributional derivatives up to order $m$ are in $L^{2}(S)$. The space $H_{0}^{m}(S)$ consists of $H^{m}(S)$ functions with vanishing trace up to order $m-1$, and the space $H^{m}(D)$ consists of $H^{m}(S)$ functions with vanishing mean. The corresponding vector Hilbert spaces are given by $\boldsymbol{H}^{m}(\Omega):=\left[H^{m}(\Omega)\right]^{2}$ and $\boldsymbol{H}_{0}^{m}(\Omega):=\left[H_{0}^{m}(\Omega)\right]^{2}$. The divergence of a vector valued function $\boldsymbol{v}=\left(v_{1}, v_{2}\right)^{t}$ is defined as $\operatorname{div} \boldsymbol{v}=\partial v_{1} / \partial x_{1}+\partial v_{2} / \partial x_{2}$ and the curl operator applied to a scalar function is defined as $\operatorname{curl} w=\left(\partial w / \partial x_{2},-\partial w / \partial x_{1}\right)^{t}$. The corresponding Hilbert spaces are given by

$$
\begin{aligned}
\boldsymbol{H}(\operatorname{div} ; \Omega): & =\left\{\boldsymbol{v} \in \boldsymbol{L}^{2}(\Omega): \operatorname{div} \boldsymbol{v} \in L^{2}(\Omega)\right\} \\
\boldsymbol{H}^{1}(\operatorname{div} ; \Omega): & =\left\{\boldsymbol{v} \in \boldsymbol{H}^{1}(\Omega): \operatorname{div} \boldsymbol{v} \in H^{1}(\Omega)\right\}
\end{aligned}
$$


Let $\mathcal{T}_{h}$ be a shape-regular, simplicial triangulation of $\Omega[12,7]$ with $h_{T}=\operatorname{diam}(T)$ for all $T \in \mathcal{T}_{h}$ and $h=\max _{T \in \mathcal{T}_{h}} h_{T}$. We denote by $\mathcal{E}_{h}$ the set of edges in the triangulation $\mathcal{T}_{h}$ and by $\mathcal{V}_{h}$ the set of vertices. Given a triangle $T \in \mathcal{T}_{h}$, we let $\mathcal{E}_{h}(T)$ be the set of three edges of $T$ and let $\mathcal{V}_{h}(T)$ be

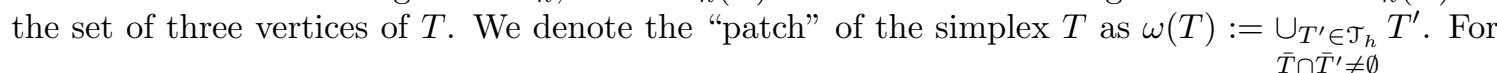
given $T \in \mathcal{T}_{h}$ we let $\boldsymbol{n}_{T}$ denote the outward unit normal of $\partial T$. When there is no ambiguity we simply write $\boldsymbol{n}$, and if we want to emphasize that $\boldsymbol{n}$ is normal to an edge $e \subset \partial T$, we write $\boldsymbol{n}_{e}$. The unit tangent of $\partial T$ obtained by rotating $\boldsymbol{n} 90$ degrees counterclockwise is denoted by $\boldsymbol{t}_{T}$. Again, when there is no confusion, we simply write $\boldsymbol{t}$.

For a simplex $S \subset \Omega$ and non-negative integer $m$, we denote by $\mathcal{P}_{m}(S)$ the set of scalar polynomials of degree less than or equal to $m$ with domain $S$. The corresponding set of vector polynomials is defined as $\mathcal{P}_{m}(S)=\left[\mathcal{P}_{m}(S)\right]^{2}$.

We end this section by stating some well-known inverse and trace inequalities $[7,12]$ that will be used extensively in the analysis. In addition, we state a local surjective property of the divergence operator on vector polynomials shown by Vogelius [25]. Below and throughout the paper, the letter $C$ denotes a generic positive constant independent of $h$ that may take different values throughout the paper.

Lemma 2.1. Let $k, m$ and $p$ be integers satisfying $0 \leq p \leq m$ and $k \geq 0$. Then there exists a constant $C>0$ such that

$$
\|v\|_{H^{m}(T)} \leq C h_{T}^{p-m}\|v\|_{H^{p}(T)} \quad \forall v \in \mathcal{P}_{k}(T), \forall T \in \mathcal{T}_{h} .
$$

Lemma 2.2. For any simply connected domain $S$ with piecewise smooth boundary $\partial S$, there exists a constant $C>0$ such that

$$
\|v\|_{L^{2}(\partial S)} \leq C\|v\|_{L^{2}(S)}^{1 / 2}\|v\|_{H^{1}(S)}^{1 / 2} \quad \forall v \in H^{1}(S) .
$$

Hence by scaling, we obtain the following estimates on any element $T \in \mathcal{T}_{h}$ :

$$
\|v\|_{L^{2}(\partial T)}^{2} \leq C\left(h_{T}^{-1}\|v\|_{L^{2}(T)}^{2}+h_{T}\|v\|_{H^{1}(T)}^{2}\right) .
$$

Lemma 2.3 (Lemma 2.5 in [25]). Let $T \in \mathcal{T}_{h}$ and $k$ be a positive integer. Then for any $q \in \mathcal{P}_{k-1}(T)$ with $q(a)=0$ for all $a \in \mathcal{V}_{h}(T)$ and $\int_{T} q d x=0$, there exists $\boldsymbol{v} \in \mathcal{P}_{k}(T) \cap \boldsymbol{H}_{0}^{1}(T)$ such that $\operatorname{div} \boldsymbol{v}=q$ and $\|\boldsymbol{v}\|_{H^{1}(T)} \leq C\|q\|_{L^{2}(T)}$.

\section{A family of $\boldsymbol{H}^{1}(\Omega) \times L^{2}(\Omega)$ conforming finite elements.}

3.1. The finite element spaces without boundary conditions. To describe the first family of Stokes elements, we must define the corresponding $H^{2}(\Omega)$ finite element space and its degrees of freedom. We denote by $\Sigma_{h}$ the $C^{1}$ finite element space consisting of piecewise polynomials of degree $(k+1)$ that are continuous at the following values (cf. Figure 3.1$)$ :

$$
\begin{array}{clll}
D^{\alpha} z(a) \quad \forall|\alpha| \leq 2, \quad \forall a \in \mathcal{V}_{h}, \quad \int_{e} \frac{\partial z}{\partial n_{e}} p d s & \forall p \in \mathcal{P}_{k-4}(e), \quad \forall e \in \mathcal{E}_{h}, \\
\int_{e} z q d s \quad \forall q \in \mathcal{P}_{k-5}(e), \forall e \in \mathcal{E}_{h}, \quad \int_{T} z r d x & \forall r \in \mathcal{P}_{k-5}(T), \quad \forall T \in \mathcal{T}_{h} .
\end{array}
$$

In the case $k=4$ the last two sets of degrees of freedom are omitted. 


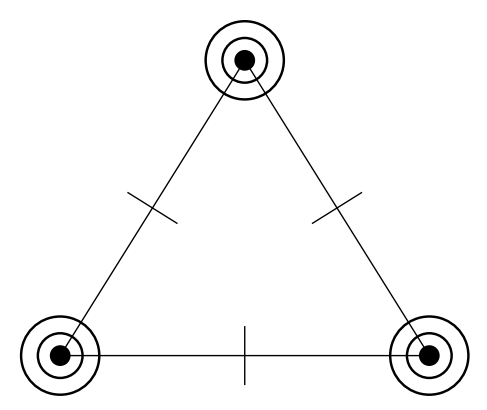

FIG. 3.1. The degrees of freedom of the lowest order $(k=4)$ Argyris element. Here, solid circles indicate function evaluation, larger circles indicate first and second derivative evaluation, and lines indicate the mean of the normal derivative.
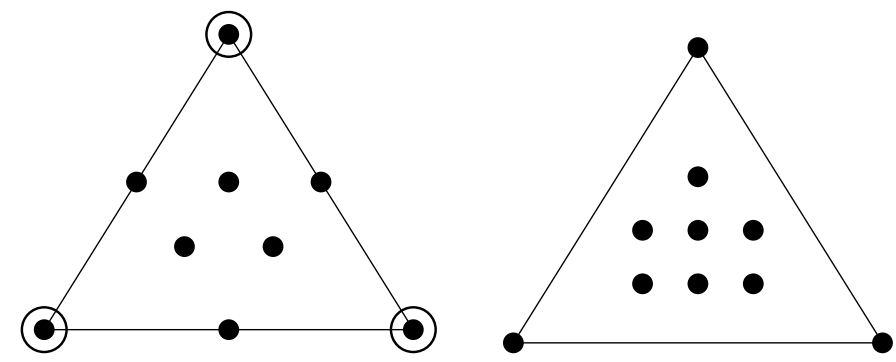

FIG. 3.2. The degrees of freedom of $\boldsymbol{V}(T)$ (left) and $Q(T)$ (right) in the case $k=4$.

Let $\boldsymbol{V}_{h}$ be the space of Hermite vector polynomials of degree $k \geq 4[12,7]$. This space consists of globally continuous piecewise polynomials whose degrees of freedom are given by (cf. Figure 3.2)

$$
\begin{aligned}
& D^{\alpha} \boldsymbol{v}(a) \quad \forall|\alpha| \leq 1, \forall a \in \mathcal{V}_{h}, \quad \int_{e} \boldsymbol{v} \cdot \boldsymbol{q} d s \quad \forall \boldsymbol{q} \in \mathcal{P}_{k-4}(e), \forall e \in \mathcal{E}_{h}, \\
& \int_{T} \boldsymbol{v} \cdot \boldsymbol{r} d x \quad \forall \boldsymbol{r} \in \mathcal{P}_{k-3}(T), \forall T \in \mathcal{T}_{h} .
\end{aligned}
$$

The pressure space $Q_{h}$ consists of piecewise polynomials of degree $(k-1)$ that are continuous at the vertices. A set of degrees of freedom that uniquely defines such a space are given as follows:

$$
p(a) \quad \forall a \in \mathcal{V}_{h}, \quad \int_{T} q r d x \quad \forall r \in \mathcal{P}_{k-1}(T) \text { with } r(a)=0, \forall a \in \mathcal{V}_{h}(T) .
$$

It is simple to check that the degrees of freedom given above form a unisolvent set for the finite element spaces $\Sigma_{h}, \boldsymbol{V}_{h}$ and $Q_{h}$. Moreover, the inclusions curl $\Sigma_{h} \subset \boldsymbol{V}_{h}$ and $\operatorname{div} \boldsymbol{V}_{h} \subset Q_{h}$ hold. To show that the first discrete complex in (1.5) is exact, it suffices to show that $\operatorname{dim} \Sigma_{h}+\operatorname{dim} Q_{h}=$ $\operatorname{dim} \boldsymbol{V}_{h}-1$.

From the degrees of freedom (3.2)-(3.3), one sees that $\operatorname{dim} \Sigma_{h}=6 V+(2 k-7) E+\frac{1}{2}(k-3)(k-4) T$, $\operatorname{dim} \boldsymbol{V}_{h}=2\left(3 V+(k-3) E+\frac{1}{2}(k-1)(k-2) T\right)$, and $\operatorname{dim} Q_{h}=V+\left(\frac{k}{2}(k+1)-3\right) T$, where $V, E$ and $T$ denote the number of vertices, edges, and triangles, respectively, in the triangulation of $\Omega$. 
Hence, $\operatorname{dim} \Sigma_{h}+\operatorname{dim} Q_{h}-\operatorname{dim} \boldsymbol{V}_{h}=V+T-E=1$ by Euler's formula. It then follows that the first sequence in (1.5) is exact. In particular, for every $q \in Q_{h}$ there exists $\boldsymbol{v} \in \boldsymbol{V}_{h}$ such that $\operatorname{div} \boldsymbol{v}=q$. The stability of such a construction is established in the next lemma.

Lemma 3.1. For every $q \in Q_{h}$ there exists $\boldsymbol{v} \in \boldsymbol{V}_{h}$ such that $\operatorname{div} \boldsymbol{v}=q$. Moreover, there exists a constant $C>0$ independent of $h$ such that $\|\boldsymbol{v}\|_{H^{1}(\Omega)} \leq C\|q\|_{L^{2}(\Omega)}$.

Proof. Let $\boldsymbol{w} \in \boldsymbol{H}^{1}(\Omega)$ satisfy $\operatorname{div} \boldsymbol{w}=q$ and $\|\boldsymbol{w}\|_{H^{1}(\Omega)} \leq C\|q\|_{L^{2}(\Omega)}$, and let $\boldsymbol{w}_{h} \in \mathcal{P}_{k}$ denote the Scott-Zhang interpolant of $\boldsymbol{w}$ [23]. We then define $\boldsymbol{v}_{1}=\left(v_{1,1}, v_{1,2}\right)^{t} \in \boldsymbol{V}_{h}$ by the following set of conditions:

$$
\boldsymbol{v}_{1}(a)=\boldsymbol{w}_{h}(a), \quad \frac{\partial v_{1, i}}{\partial x_{i}}(a)=\frac{1}{2} q(a), \quad \frac{\partial v_{1, i}}{\partial x_{j}}(a)=0(i \neq j)
$$

for all vertices $a \in \mathcal{V}_{h}$;

$$
\int_{e} \boldsymbol{v}_{1} \cdot \boldsymbol{q} d s=\int_{e} \boldsymbol{w} \cdot \boldsymbol{q} d s \quad \forall \boldsymbol{q} \in \mathcal{P}_{k-4}(e)
$$

for all edges $e \in \mathcal{E}_{h}$;

$$
\int_{T} \boldsymbol{v}_{1} \cdot \boldsymbol{r} d x=\int_{T} \boldsymbol{w} \cdot \boldsymbol{r} d x \quad \forall \boldsymbol{r} \in \mathcal{P}_{k-3}(T)
$$

for all triangles $T \in \mathcal{T}_{h}$. We clearly have $\operatorname{div} \boldsymbol{v}_{1}(a)=q(a)$ at all the vertices. Moreover, by integration by parts and (3.4) we have

$$
\int_{T} \operatorname{div} \boldsymbol{v}_{1} d s=\int_{\partial T} \boldsymbol{v}_{1} \cdot \boldsymbol{n} d s=\int_{\partial T} \boldsymbol{w} \cdot \boldsymbol{n} d s=\int_{T} \operatorname{div} \boldsymbol{w} d x=\int_{T} q d x
$$

Thus, $\left(q-\operatorname{div} \boldsymbol{v}_{1}\right) \in Q_{h}$ vanishes at all of the vertices and has vanishing mean on each triangle. Therefore by Lemma 2.3, for each $T \in \mathcal{T}_{h}$, there exists $\boldsymbol{v}_{2, T} \in \mathcal{P}_{k}(T)$ such that $\left.\operatorname{div} \boldsymbol{v}_{2, T}\right|_{T}=$ $\left.\left(q-\operatorname{div} \boldsymbol{v}_{1}\right)\right|_{T},\left.\boldsymbol{v}_{2, T}\right|_{\partial T}=0$ and $\left\|\boldsymbol{v}_{2, T}\right\|_{H^{1}(T)} \leq C\left\|q-\operatorname{div} \boldsymbol{v}_{1}\right\|_{L^{2}(T)} \leq C\left(\|q\|_{L^{2}(T)}+\left\|\boldsymbol{v}_{1}\right\|_{H^{1}(T)}\right)$. We then define $\boldsymbol{v}_{2}$ by $\left.\boldsymbol{v}_{2}\right|_{T}=\left.\boldsymbol{v}_{2, T}\right|_{T} \forall T \in \mathcal{T}_{h}$. The function $\boldsymbol{v}_{2}$ is $C^{1}$ at the vertices of the triangulation since $\left.\nabla \boldsymbol{v}_{2, T}\right|_{T}(a)=0$ for all $a \in \mathcal{V}_{h}(T)$. Thus, $\boldsymbol{v}_{2} \in \boldsymbol{V}_{h}$. Setting $\boldsymbol{v}=\boldsymbol{v}_{1}+\boldsymbol{v}_{2}$, we have $\operatorname{div} \boldsymbol{v}=q$ and $\|\boldsymbol{v}\|_{H^{1}(\Omega)} \leq\left\|\boldsymbol{v}_{1}\right\|_{H^{1}(\Omega)}+\left\|\boldsymbol{v}_{2}\right\|_{H^{1}(\Omega)} \leq C\left(\|q\|_{L^{2}(\Omega)}+\left\|\boldsymbol{v}_{1}\right\|_{H^{1}(\Omega)}\right)$. Thus, it remains to show $\left\|\boldsymbol{v}_{1}\right\|_{H^{1}(\Omega)} \leq C\|q\|_{L^{2}(\Omega)}$.

Set $\boldsymbol{z}=\boldsymbol{v}_{1}-\boldsymbol{w}_{h}$ and $\hat{\boldsymbol{z}}(\hat{x})=\boldsymbol{z}(x)$, where $x=F_{T}(\hat{x})$ and $F_{T}(\hat{x})=A \hat{x}+b$ is the affine mapping from $\hat{T}$ to $T$. Here, $\hat{T}$ denotes the reference triangle with vertices $(0,0),(1,0)$ and $(0,1)$. Since the degrees of freedom (3.2) form a unisolvent set over $\mathcal{P}_{k}(T)$, and since all norms are equivalent in a finite dimensional setting, we have $(m \geq 0)$

$$
\|\hat{\boldsymbol{z}}\|_{H^{m}(\hat{T})} \approx \sum_{\hat{a} \in \mathcal{V}_{h}(\hat{T})}|\hat{\nabla} \hat{\boldsymbol{z}}(\hat{a})|^{2}+\sum_{\hat{e} \in \mathcal{E}_{h}(\hat{T})}\left|\sup _{\substack{\hat{\boldsymbol{q}} \in \mathcal{P}_{k-4}(\hat{T}) \\\|\hat{\boldsymbol{q}}\|_{L^{2}(\hat{e})}=1}} \int_{\hat{e}} \hat{\boldsymbol{z}} \cdot \hat{\boldsymbol{q}} d \hat{s}\right|^{2}+\left|\sup _{\substack{\hat{\boldsymbol{r}} \in \mathcal{P}_{k-3}(\hat{T}) \\\|\hat{\boldsymbol{r}}\|_{L^{2}(\hat{T})}=1}} \int_{\hat{T}} \hat{\boldsymbol{z}} \cdot \hat{\boldsymbol{r}} d \hat{x}\right|^{2},
$$

where we have used the fact that $\hat{\boldsymbol{z}}$ vanishes at the vertices of $\hat{T}$. By standard scaling arguments, we have

$$
\sum_{\hat{a} \in \mathcal{V}_{h}(\hat{T})}|\hat{\nabla} \hat{\boldsymbol{z}}(\hat{a})|^{2} \leq C h_{T}^{2} \sum_{a \in \mathcal{V}_{h}(T)}\left|\nabla\left(\boldsymbol{v}_{1}-\boldsymbol{w}_{h}\right)(a)\right|^{2} \leq C\left(\|q\|_{L^{2}(T)}^{2}+\left\|\boldsymbol{w}_{h}\right\|_{H^{1}(T)}^{2}\right) .
$$


Next, let $\hat{\boldsymbol{r}}_{*} \in \boldsymbol{P}_{k-3}(\hat{T})$ be a function satisfying $\left\|\hat{\boldsymbol{r}}_{*}\right\|_{L^{2}(\hat{T})}=1$ and

$$
\sup _{\substack{\hat{\boldsymbol{r}} \in \mathcal{P}_{k-3}(\hat{T}) \\\|\hat{\boldsymbol{r}}\|_{L^{2}(\hat{T})}=1}} \int_{\hat{T}} \hat{\boldsymbol{z}} \cdot \hat{\boldsymbol{r}} d \hat{x}=\int_{\hat{T}} \hat{\boldsymbol{z}} \cdot \hat{\boldsymbol{r}}_{*} d \hat{x}
$$

Set $\boldsymbol{r}_{*}(x)=\left|J_{F}\right|^{-1 / 2} \hat{\boldsymbol{r}}_{*}(\hat{x})$, where $\left|J_{F}\right|$ is the determinant of the Jacobian of $F$, i.e, $\left|J_{F}\right|=\operatorname{det}(A)=$ $2|T|$. Then $\left\|\boldsymbol{r}_{*}\right\|_{L^{2}(T)}=1$ and by (3.4) we have

$$
\begin{aligned}
\left|\sup _{\hat{\boldsymbol{r}} \in \mathcal{P}_{k-3}(\hat{T})} \int_{\hat{T}} \hat{\boldsymbol{z}} \cdot \hat{\boldsymbol{r}} d \hat{x}\right|^{2} & =\left|J_{F}\right|^{-1}\left|\int_{T} \boldsymbol{z} \cdot \boldsymbol{r}_{*} d x\right|^{2} \\
& \leq C h_{T}^{-2}\left|\sup _{\substack{\boldsymbol{r} \in \mathcal{P}_{k-3}(T) \\
\|\boldsymbol{r}\|_{L^{2}(T)}}} \int_{T}\left(\boldsymbol{w}-\boldsymbol{w}_{h}\right) \cdot \boldsymbol{r} d x\right|^{2} \leq C h_{T}^{-2}\left\|\boldsymbol{w}-\boldsymbol{w}_{h}\right\|_{L^{2}(T)}^{2}
\end{aligned}
$$

Similar arguments show

$$
\sum_{\hat{e} \in \mathcal{E}_{h}(\hat{T})}\left|\sup _{\substack{\hat{\boldsymbol{q}} \in \mathcal{P}_{k-4}(\hat{T}) \\\|\hat{\boldsymbol{q}}\|_{L^{2}(\hat{e})}=1}} \int_{\hat{e}} \hat{\boldsymbol{z}} \cdot \hat{\boldsymbol{q}} d \hat{s}\right|^{2} \leq C h_{T}^{-1}\left\|\boldsymbol{w}-\boldsymbol{w}_{h}\right\|_{L^{2}(\partial T)}^{2} .
$$

Applying the estimates (3.6) and (3.8)-(3.9) to (3.5) and using the approximation properties of $\boldsymbol{w}_{h}[23]$ we obtain

$$
\begin{aligned}
\|\hat{\boldsymbol{z}}\|_{H^{m}(\hat{T})} & \leq C\left(\|q\|_{L^{2}(T)}^{2}+\left\|\boldsymbol{w}_{h}\right\|_{H^{1}(T)}^{2}+h_{T}^{-2}\left\|\boldsymbol{w}-\boldsymbol{w}_{h}\right\|_{L^{2}(T)}^{2}+h_{T}^{-1}\left\|\boldsymbol{w}-\boldsymbol{w}_{h}\right\|_{L^{2}(\partial T)}^{2}\right) \\
& \leq C\left(\|q\|_{L^{(T)}}^{2}+\|\boldsymbol{w}\|_{H^{1}(\omega(T))}^{2}\right) .
\end{aligned}
$$

Therefore by scaling,

$$
\left\|\boldsymbol{v}_{1}-\boldsymbol{w}_{h}\right\|_{H^{1}(T)}^{2} \leq C\left(|\hat{\boldsymbol{z}}|_{H^{1}(\hat{T})}^{2}+h_{T}^{2}\|\hat{\boldsymbol{z}}\|_{L^{2}(\hat{T})}^{2}\right) \leq C\left(\|\boldsymbol{w}\|_{H^{1}(\omega(T))}^{2}+\|q\|_{L^{2}(T)}^{2}\right) .
$$

Employing the triangle inequality and summing over $T \in \mathcal{T}_{h}$, we obtain $\left\|\boldsymbol{v}_{1}\right\|_{H^{1}(\Omega)} \leq C\left(\|\boldsymbol{w}\|_{H^{1}(\Omega)}+\right.$ $\left.\|q\|_{L^{2}(\Omega)}\right) \leq C\|q\|_{L^{2}(\Omega)}$. This completes the proof.

3.2. The finite element spaces with homogeneous boundary conditions. We now discuss the finite element spaces incorporating homogenous boundary conditions. A natural approach is to take the spaces as $\Sigma_{h, 0}=\Sigma_{h} \cap H_{0}^{2}(\Omega), \boldsymbol{V}_{h, 0}=\boldsymbol{V}_{h} \cap \boldsymbol{H}_{0}^{1}(\Omega)$ and $Q_{h, 0}=Q_{h} \cap \stackrel{\circ}{L}^{2}(\Omega)$. Here we argue that this construction does not form an exact sequence; in particular, the space $Q_{h, 0}$ is strictly larger than $\operatorname{div} \boldsymbol{V}_{h, 0}$. Therefore these two spaces do not form a stable finite element pair for the Stokes problem.

We arrive at these conclusions using a similar counting argument as in the previous section. Clearly the zero mean value restriction implies that $\operatorname{dim} Q_{h, 0}=\operatorname{dim} Q_{h}-1=V+\left(\frac{k}{2}(k+1)-3\right) T-1$. The requirement $\boldsymbol{V}_{h, 0} \subset \boldsymbol{H}_{0}^{1}(\Omega)$ can be achieved by imposing the following constraints for $\boldsymbol{v} \in \boldsymbol{V}_{h, 0}$ : (i) $\boldsymbol{v}=0$ and $\partial \boldsymbol{v} / \partial \boldsymbol{t}=0$ at all boundary vertices; (ii) the $(k-4)$ th order moments of $\boldsymbol{v}$ vanish on each boundary edge. Note that $\nabla \boldsymbol{v}=0$ at all corner vertices of the triangulation. A corner vertex is a boundary vertex such that the two adjacent boundary edges do not lie on a straight line. Denote by $V_{0}$ the number of interior vertices, $E_{0}$ the number of interior edges, and $V_{b}$ the number 
of boundary vertices not including corner vertices in the triangulation. We then find $\operatorname{dim} \boldsymbol{V}_{h, 0}=$ $2\left(3 V_{0}+(k-3) E_{0}+\frac{1}{2}(k-1)(k-2) T+V_{b}\right)$. Lastly, we construct the space $\Sigma_{h, 0}$ by requiring all $z \in \Sigma_{h, 0}$ to satisfy the following properties: (i) $z=0$ and $\nabla z=0$ for all boundary vertices; (ii) the $(k-5)$ th order moments of $z$ and the $(k-4)$ th order moments of $\partial z / \partial n$ vanish on each boundary edge; (iii) the second tangental derivative $\partial^{2} z / \partial s^{2}$ and the mixed tangental-normal derivative $\partial^{2} z / \partial s \partial n$ vanish at all boundary vertices. We then have $\operatorname{dim} \Sigma_{h, 0}=6 V_{0}+(2 k-7) E_{0}+\frac{1}{2}(k-3)(k-4) T+V_{b}$.

Since curl $\Sigma_{h, 0} \subset \boldsymbol{V}_{h, 0}$ and $\operatorname{div} \boldsymbol{V}_{h, 0} \subset Q_{h, 0}$, it is easy to see that the sequence

$$
0 \stackrel{\subset}{\longrightarrow} \Sigma_{h, 0} \stackrel{\text { curl }}{\longrightarrow} \boldsymbol{V}_{h, 0} \stackrel{\text { div }}{\longrightarrow} Q_{h, 0} \longrightarrow 0
$$

will be exact provided $\operatorname{dim} \Sigma_{h, 0}+\operatorname{dim} Q_{h, 0}=\operatorname{dim} \boldsymbol{V}_{h, 0}$. However, using the identities $T+V-E=1$ and $E-E_{0}=V-V_{0}$, we find

$$
\begin{aligned}
\operatorname{dim} \Sigma_{h, 0}+\operatorname{dim} Q_{h, 0}-\operatorname{dim} \boldsymbol{V}_{h, 0}= & 6 V_{0}+(2 k-7) E_{0}+\frac{1}{2}(k-3)(k-4) T+V_{b} \\
& +V+\left(\frac{k}{2}(k+1)-3\right) T-1 \\
& \quad-\left(2\left(3 V_{0}+(k-3) E_{0}+\frac{1}{2}(k-1)(k-2) T+V_{b}\right)\right. \\
= & T+V-1-E_{0}-V_{b}=E-E_{0}-V_{b} \\
= & V-V_{0}-V_{b}=V_{c},
\end{aligned}
$$

where $V_{c}$ denotes the number of corner vertices in the triangulation. Above, we have used the identity $V=V_{0}+V_{b}+V_{c}$. Since $V_{c}>0$, we clearly see that the spaces $\Sigma_{h, 0}, V_{h, 0}$ and $Q_{h, 0}$ constructed above do not form an exact sequence. In fact, this is easy to see without relying on the counting argument above. Indeed, since $\boldsymbol{v} \in \boldsymbol{V}_{h, 0}$ has vanishing derivatives at the corners of the domain, the divergence of $\boldsymbol{v}$ vanishes at these points as well. Since no restriction is imposed on $Q_{h, 0}$ at the corner points, the sequence cannot be exact.

We now discuss an alternative construction of the finite element spaces $\Sigma_{h, 0}, \boldsymbol{V}_{h, 0}, Q_{h, 0}$. The idea is to modify the spaces constructed above on triangles with corner vertices. To construct these spaces, we make the following assumption about the mesh:

(M) Every corner vertex is non-singular.

We recall a boundary vertex is singular if all the edges meeting at this vertex fall on two straight lines (see [22] and [25] for details). It is easy to see that assumption (M) is equivalent to the assumption that no triangle has more than one boundary edge.

We define the finite element spaces with homogenous boundary conditions as

$$
\begin{aligned}
\Sigma_{h, 0} & =\left\{z \in H_{0}^{2}(\Omega):\left.z\right|_{T} \in \Sigma_{h}(T), z \text { is } C^{2} \text { at all non-corner vertices }\right\} \\
\boldsymbol{V}_{h, 0} & =\left\{\boldsymbol{v} \in \boldsymbol{H}_{0}^{1}(\Omega):\left.\boldsymbol{v}\right|_{T} \in \boldsymbol{V}(T), \boldsymbol{v} \text { is } C^{1} \text { at all non-corner vertices }\right\}, \\
Q_{h, 0} & =\left\{q \in \stackrel{\circ}{L}^{2}(\Omega):\left.q\right|_{T} \in Q(T), q \text { is } C^{0} \text { at all non-corner vertices }\right\} .
\end{aligned}
$$

REMARK 3.2. If the corner vertices are all singular then the finite element spaces (3.10) are the same as those considered earlier.

Denote by $E_{c, 0}$ the number of interior edges that have a corner vertex as one of its endpoints. Then by following similar arguments discussed above, we have $\operatorname{dim} \boldsymbol{V}_{h, 0}=2\left(3 V_{0}+(k-3) E_{0}+\frac{1}{2}(k-\right.$ 1) $\left.(k-2) T+V_{b}+E_{c, 0}\right)$, and $\operatorname{dim} Q_{h, 0}=v+\left(\frac{k}{2}(k-1)-3\right) T-1+E_{c, 0}$. Furthermore, using the 
arguments above and arguments found in [22, Section 6], we find $\operatorname{dim} \Sigma_{h, 0}=6 V_{0}+(2 k-7) E_{0}+$ $\frac{1}{2}(k-4)(k-3) T+V_{b}+E_{c, 0}-V_{c}$. It is then an easy exercise to show $\operatorname{dim} \Sigma_{h, 0}+\operatorname{dim} Q_{h, 0}=\operatorname{dim} \boldsymbol{V}_{h, 0}$, implying the corresponding sequence is exact.

We also have the following stability result, analogous to Lemma 3.1.

Lemma 3.3. Suppose that assumption (M) holds, and let $\boldsymbol{V}_{h, 0}$ and $Q_{h, 0}$ be defined by (3.10b) and (3.10c) respectively. Then for any $q \in Q_{h, 0}$, there exists $a \boldsymbol{v} \in \boldsymbol{V}_{h, 0}$ such that $\operatorname{div} \boldsymbol{v}=q$ and $\|\boldsymbol{v}\|_{H^{1}(\Omega)} \leq C\|q\|_{L^{2}(\Omega)}$. Consequently the LBB condition

$$
C\|q\|_{L^{2}(\Omega)} \leq \sup _{\boldsymbol{v} \in \boldsymbol{V}_{h, 0} \backslash\{0\}} \frac{\int_{\Omega}(\operatorname{div} \boldsymbol{v}) q d x}{\|\boldsymbol{v}\|_{H^{1}(\Omega)}} \quad \forall q \in Q_{h, 0}
$$

holds for a constant $C>0$ independent of $h$.

Proof. The proof strongly resembles the proof of Lemma 3.1. As before we let $\boldsymbol{w} \in \boldsymbol{H}_{0}^{1}(\Omega)$ satisfy $\operatorname{div} \boldsymbol{w}=q$ and $\|\boldsymbol{w}\|_{H^{1}(\Omega)} \leq C\|q\|_{L^{2}(\Omega)}\left[15\right.$, Corollary 2.4], and let $\boldsymbol{w}_{h} \in \mathcal{P}_{k} \cap \boldsymbol{H}_{0}^{1}(\Omega)$ denote the Scott-Zhang interpolant of $\boldsymbol{w}$. We then define $\boldsymbol{v}_{1} \in \boldsymbol{V}_{h, 0}$ as follows. At all vertices in the triangulation, we set $\boldsymbol{v}(a)=\boldsymbol{w}_{h}(a)$, and at all interior vertices of the triangulation we set

$$
\frac{\partial v_{1, i}}{\partial x_{i}}(a)=\frac{1}{2} q(a), \quad \frac{\partial v_{1, i}}{\partial x_{j}}(a)=0(i \neq j) .
$$

For all edges $e \in \mathcal{E}_{h}$, we impose the condition

$$
\int_{e} \boldsymbol{v}_{1} \cdot \boldsymbol{q} d s=\int_{e} \boldsymbol{w} \cdot \boldsymbol{q} d s \quad \forall \boldsymbol{q} \in \mathcal{P}_{k-4}(e),
$$

and for all $T$, we specify that

$$
\int_{T} \boldsymbol{v}_{1} \cdot \boldsymbol{r} d x=\int_{T} \boldsymbol{w} \cdot \boldsymbol{r} d x \quad \forall \boldsymbol{r} \in \mathcal{P}_{k-3}(T) .
$$

Note that at this point, $\boldsymbol{v}_{1}$ is uniquely determined in interior triangles, i.e., triangles $T \in \mathcal{T}_{h}$ that satisfy $\partial T \cap \partial \Omega=\emptyset$. Moreover we have $\operatorname{div} \boldsymbol{v}_{1}(a)=q(a)$ at all interior vertices and $\int_{T} \operatorname{div} \boldsymbol{v}_{1} d x=$ $\int_{T} q d x$ for all $T \in \mathcal{T}_{h}$. Furthermore, by the proof of Lemma 3.1, we have $\|\boldsymbol{v}\|_{H^{1}(T)} \leq C\left(\|q\|_{L^{2}(T)}+\right.$ $\left.\|\boldsymbol{w}\|_{H^{1}(\omega(T))}\right)$ for all interior triangles $T$.

Next, let $a$ be a non-corner boundary vertex, and denote by $\boldsymbol{t}$ a unit vector parallel to the boundary of $\Omega$ at $a$. Setting $\frac{\partial \boldsymbol{v}_{1}}{\partial \boldsymbol{t}}(a)=0$, we have

$$
\operatorname{div} \boldsymbol{v}(a)=n_{1} \frac{\partial v_{1,1}}{\partial \boldsymbol{n}}(a)+n_{2} \frac{\partial v_{1,2}}{\partial \boldsymbol{n}}(a)=\boldsymbol{n} \cdot \frac{\partial \boldsymbol{v}}{\partial \boldsymbol{n}}(a) .
$$

where $\boldsymbol{n}=\left(n_{1}, n_{2}\right)^{t}$ denotes the outward unit normal of $\partial \Omega$ at $a$. We then prescribe

$$
\frac{\partial \boldsymbol{v}}{\partial \boldsymbol{n}}(a)=\boldsymbol{n} q(a)
$$

Let $a$ be a corner vertex and denote by $\left\{T_{i}\right\}_{i=1}^{N}$ the triangles that have $a$ as a vertex (cf. Figure 3.3). Without loss of generality, we assume that the triangles are labeled in a counterclockwise fashion such that $T_{1}$ and $T_{N}$ each have one edge that intersects the boundary $\partial \Omega$. Let $\left\{e_{i}\right\}_{i=1}^{N+1}$ be the edges in $\mathcal{E}_{h}$ such that $a \subset \bar{e}$, again, labeled in a counterclockwise fashion, and denote by $\boldsymbol{t}_{i}$ 
a unit vector parallel to edge $e_{i}$. Let $\boldsymbol{\eta}^{\perp}=\left(\eta_{2},-\eta_{1}\right)^{t}$. Since $\boldsymbol{t}_{i}$ and $\boldsymbol{t}_{i+1}$ are linearly independent vectors, we can write grad $\boldsymbol{v}_{1}$ as a linear combination of $\boldsymbol{t}_{i}^{\perp}$ and $\boldsymbol{t}_{i+1}^{\perp}$, whose coefficients depend on $\partial \boldsymbol{v}_{1} / \partial \boldsymbol{t}_{i}$ and $\partial \boldsymbol{v}_{1} / \partial \boldsymbol{t}_{i+1}$. We then easily find

$$
\left.\operatorname{div} \boldsymbol{v}_{1}\right|_{T_{i}}=\left(\boldsymbol{t}_{i+1}^{\perp} \cdot \frac{\partial \boldsymbol{v}_{1}}{\partial \boldsymbol{t}_{i}}-\boldsymbol{t}_{i}^{\perp} \cdot \frac{\partial \boldsymbol{v}_{1}}{\partial \boldsymbol{t}_{i+1}}\right) /\left(\boldsymbol{t}_{i} \cdot \boldsymbol{t}_{i+1}^{\perp}\right) .
$$

Consider the case $N=2$. To satisfy the homogenous boundary conditions, we set $\partial \boldsymbol{v}_{1} /\left.\partial \boldsymbol{t}_{1}\right|_{T_{1}}(a)$ $=\partial \boldsymbol{v}_{1} /\left.\partial \boldsymbol{t}_{3}\right|_{T_{N}}(a)=0$. We then have

$$
\left.\operatorname{div} \boldsymbol{v}_{1}\right|_{T_{1}}(a)=-\left(\boldsymbol{t}_{1}^{\perp} \cdot \frac{\partial \boldsymbol{v}_{1}}{\partial \boldsymbol{t}_{2}}(a)\right) /\left(\boldsymbol{t}_{1} \cdot \boldsymbol{t}_{2}^{\perp}\right)
$$

and

$$
\left.\operatorname{div} \boldsymbol{v}_{1}\right|_{T_{2}}(a)=\left(\boldsymbol{t}_{3}^{\perp} \cdot \frac{\partial \boldsymbol{v}_{1}}{\partial \boldsymbol{t}_{2}}(a)\right) /\left(\boldsymbol{t}_{2} \cdot \boldsymbol{t}_{3}^{\perp}\right)
$$

Prescribing

$$
\frac{\partial \boldsymbol{v}_{1}}{\partial \boldsymbol{t}_{2}}(a)=\frac{\boldsymbol{t}_{1} \cdot \boldsymbol{t}_{2}^{\perp}}{\boldsymbol{t}_{3}^{\perp} \cdot \boldsymbol{t}_{1}} \boldsymbol{t}_{3} c_{1}+\frac{\boldsymbol{t}_{2} \cdot \boldsymbol{t}_{3}^{\perp}}{\boldsymbol{t}_{3}^{\perp} \cdot \boldsymbol{t}_{1}} \boldsymbol{t}_{1} c_{2}
$$

for some constants $c_{1}, c_{2}$, we have $\left.\operatorname{div} \boldsymbol{v}\right|_{T_{i}}(a)=c_{i}(i=1,2)$. In particular, by choosing $c_{i}=\left.q\right|_{T_{i}}(a)$, we have $\left.\operatorname{div} \boldsymbol{v}\right|_{T_{i}}(a)=\left.q\right|_{T_{i}}(a)$.

In the general case $N \geq 2$, we can repeat this procedure for all triangles in a counterclockwise fashion. Namely, using the arguments in [25, Lemma 2.6], we construct $\boldsymbol{v}_{1}$ to satisfy $\left.\operatorname{div} \boldsymbol{v}_{1}\right|_{T_{1}}(a)=$ $c_{1},\left.\operatorname{div} \boldsymbol{v}_{1}\right|_{T_{N}}(a)=c_{2 N-2}$ and $\left.\operatorname{div} \boldsymbol{v}_{1}\right|_{T_{i}}(a)=c_{2 i-2}+c_{2 i-1}(2 \leq i \leq N-1)$ for some constants $c_{i} \in \mathbb{R}$. We then choose $c_{1}=\left.q(a)\right|_{T_{1}}, c_{2 N-2}=\left.q(a)\right|_{T_{N}}$ and $c_{2 i-2}=\left.q(a)\right|_{T_{i}}$ and $c_{2 i-1}=0(2 \leq i \leq N-1)$. With this choice, we clearly have $\operatorname{div} \boldsymbol{v}_{1}(a)_{T_{i}}=\left.q(a)\right|_{T_{i}}(1 \leq i \leq N)$. Furthermore, by scaling arguments (see [25, Lemma 2.6]), we have for all boundary triangles, $\left\|\boldsymbol{v}_{1}\right\|_{H^{1}(T)} \leq C\left(\|q\|_{L^{2}(\omega(T))}+\right.$ $\left.\|\boldsymbol{w}\|_{H^{1}(\omega(T))}\right)$. It then follows that $\left\|\boldsymbol{v}_{1}\right\|_{H^{1}(\Omega)} \leq C\left(\|q\|_{L^{2}(\Omega)}+\|\boldsymbol{w}\|_{H^{1}(\Omega)}\right) \leq C\|q\|_{L^{2}(\Omega)}$.

At this point the proof is the same as the proof of Lemma 3.1. Namely, we let $\boldsymbol{v}_{2, T} \in \mathcal{P}_{k}(T) \cap$ $\boldsymbol{H}_{0}^{1}(T)$ satisfy $\operatorname{div} \boldsymbol{v}_{2, T}=\left.\left(q-\boldsymbol{v}_{1}\right)\right|_{T}$ and define $\boldsymbol{v}_{2} \in \boldsymbol{V}_{h, 0}$ by $\left.\boldsymbol{v}_{2}\right|_{T}=\left.\boldsymbol{v}_{2, T}\right|_{T}$. Then $\boldsymbol{v}:=\boldsymbol{v}_{1}+\boldsymbol{v}_{2}$ satisfies $\operatorname{div} \boldsymbol{v}=q$ and $\|\boldsymbol{v}\|_{H^{1}(\Omega)} \leq C\|q\|_{L^{2}(\Omega)}$. $\mathrm{\square}$

4. A family of $\boldsymbol{H}^{1}(\operatorname{div} ; \Omega) \times H^{1}(\Omega)$ conforming finite elements. In this section we develop a family of $\boldsymbol{H}^{1}(\operatorname{div} ; \Omega) \times H^{1}(\Omega)$ conforming finite element pairs that also produce pointwise divergence-free velocity approximations for the Stokes problem. Essentially, these spaces are obtained by removing sets of functions from the velocity and pressure spaces $\boldsymbol{V}_{h}$ and $Q_{h}$.

4.1. The finite element spaces without boundary conditions. The local pressure space is given by the $(k-3)(k+4) / 2$ dimensional (scalar) MINI space [2]

$$
S(T)=\mathcal{P}_{k-3}(T) \cup\left(\mathcal{P}_{k-1}(T) \cap H_{0}^{1}(T)\right) .
$$

Again, $k$ is an integer satisfying $k \geq 4$. A function in $S(T)$ is uniquely determined by the following values (cf. Figure 4.1)

$$
\begin{aligned}
& w(a) \quad \forall a \in \mathcal{V}_{h}(T), \quad \int_{e} w \kappa d s \quad \forall \kappa \in \mathcal{P}_{k-5}(e), \forall e \in \mathcal{E}_{h}(T), \\
& \int_{T} w \omega d x \quad \forall \omega \in \mathcal{P}_{k-4}(T) .
\end{aligned}
$$




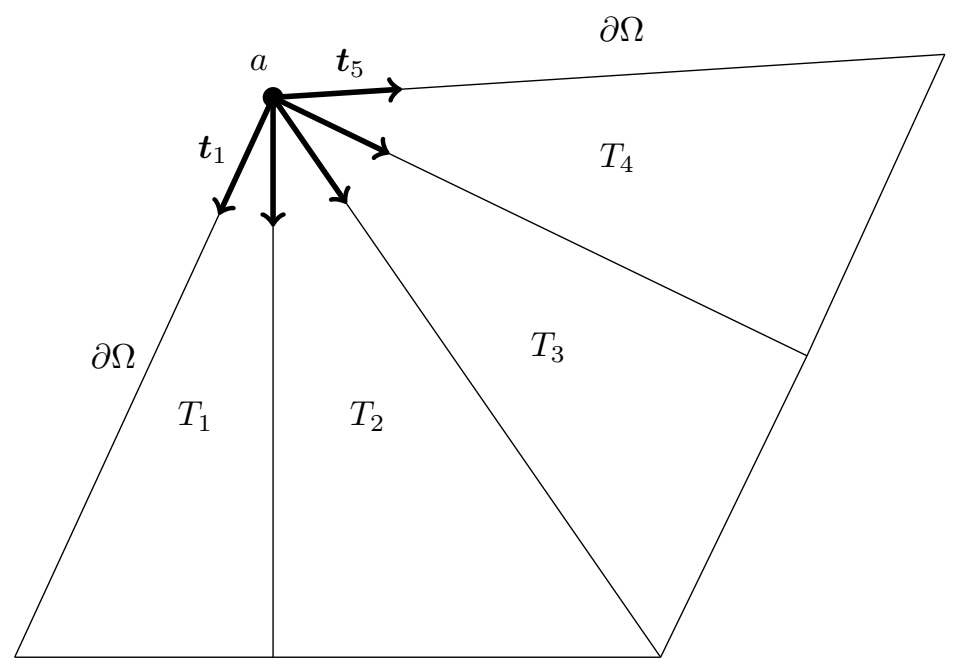

FIG. 3.3. A pictorial description of the proof of Lemma 3.3 with $N=4$.
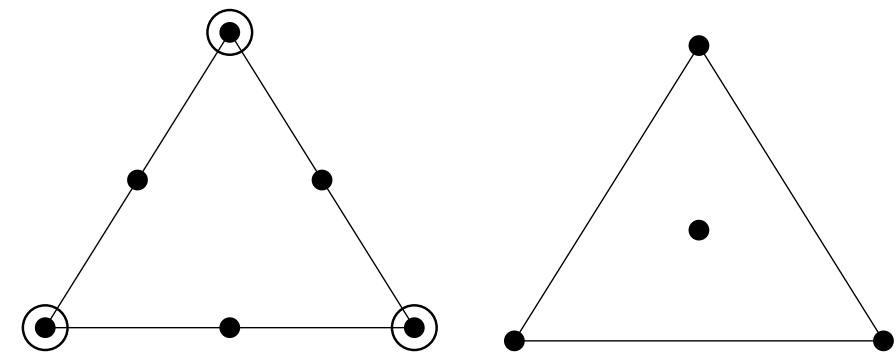

FIG. 4.1. The degrees of freedom of $\boldsymbol{W}(T)$ (left) and $S(T)$ (right) in the case $k=4$.

Lemma 4.1. The degrees of freedom (4.2) are unisolvent on $S(T)$.

Proof. Since there are exactly $\left(3+3(k-4)+\frac{1}{2}(k-2)(k-3)\right)=\frac{1}{2}(k+4)(k-3)$ degrees of freedom given in (4.2), it suffices to show that if $w \in S(T)$ vanishes at the degrees of freedom, then $w \equiv 0$. Since $\left.w\right|_{\partial T} \in \mathcal{P}_{k-3}(T)$, the first two sets of conditions in (4.2) imply that $\left.w\right|_{\partial T}=0$. Therefore we can write $w=b_{T} p$ for some $p \in \mathcal{P}_{k-4}(T)$, where $b_{T} \in H_{0}^{1}(\Omega)$ denotes the cubic bubble function. We then have $0=\int_{T} w p d x=\int_{T} b_{T} p^{2} d x$. Since $b_{T}>0$ in $T$, we conclude that $p \equiv 0$ and therefore $w \equiv 0$.

The local space of the velocity element is defined as

$$
\boldsymbol{W}(T)=\left\{\boldsymbol{v} \in \mathcal{P}_{k}(T): \operatorname{div} \boldsymbol{v} \in S(T)\right\} .
$$

It is easy to see that $\operatorname{dim} \boldsymbol{W}(T) \geq(k+2)(k+1)-\frac{1}{2}(k(k+1)-(k-3)(k+4))=k^{2}+3 k-4$. A unisolvent set of degrees of freedom for $\boldsymbol{W}(T)$ is given by

$$
D^{\alpha} \boldsymbol{v}(a) \forall|\alpha| \leq 1, \forall a \in \mathcal{V}_{h}(T), \quad \int_{e_{11}} \boldsymbol{v} \cdot \boldsymbol{\kappa} d s \quad \forall \boldsymbol{\kappa} \in \mathcal{P}_{k-4}(e), \forall e \in \mathcal{E}_{h}(T)
$$




$$
\begin{aligned}
& \int_{e}(\operatorname{div} \boldsymbol{v}) \omega d s \quad \forall \omega \in \mathcal{P}_{k-5}(e), \forall e \in \mathcal{E}_{h}(T), \\
& \int_{T} \boldsymbol{v} \cdot \boldsymbol{\phi} d x \quad \forall \phi \in \boldsymbol{R}(T):=\operatorname{grad}\left(\mathcal{P}_{k-4}(T)\right)+\operatorname{curl}\left(b_{T}^{2} \mathcal{P}_{k-5}(T)\right) .
\end{aligned}
$$

In the case $k=4$ the degrees of freedom (4.3b)-(4.3c) are omitted.

LEMma 4.2. The degrees of freedom (4.3) are unisolvent on $\boldsymbol{W}(T)$.

Proof. Similar to Lemma 4.1, we show that if $\boldsymbol{v} \in \boldsymbol{W}(T)$ vanishes at the degrees of freedom, then $\boldsymbol{v}$ is identically zero.

If $\boldsymbol{v} \in \boldsymbol{W}(T)$ vanishes on the degrees of freedom (4.3), then $\left.\operatorname{div} \boldsymbol{v}\right|_{\partial T}=0$ and $\left.\boldsymbol{v}\right|_{\partial T}=0$. We can then write $\operatorname{div} \boldsymbol{v}=b_{T} p$ for some $p \in \mathcal{P}_{k-4}(T)$. By (4.3), and integration by parts, we obtain

$$
\int_{T} b_{T} p^{2} d x=\int_{T}(\operatorname{div} \boldsymbol{v}) p d x=-\int_{T} \boldsymbol{v} \cdot \operatorname{grad} p d x+\int_{\partial T}(\boldsymbol{v} \cdot \boldsymbol{n}) p d s=0 .
$$

It then follows that $\operatorname{div} \boldsymbol{v}=0$ and therefore we may write $\boldsymbol{v}=\operatorname{curl} w$ for some $w \in \mathcal{P}_{k+1}(T)$ unique up to a constant. Since $0=\boldsymbol{v} \cdot \boldsymbol{n}=\frac{\partial w}{\partial s}$ on $\partial T$, we may assume that $\left.w\right|_{\partial T}=0$. It then follows from (4.3a) that $D^{\alpha} w(a)=0$ for all multi-indices $|\alpha| \leq 2$ and all vertices of $T$. Moreover, by (4.3a) there holds $\int_{e}\left(\partial w / \partial n_{e}\right) p=0$ for all $p \in \mathcal{P}_{k-4}(e)$. We then conclude that $\nabla w$ vanishes on $\partial T$ as well. We may then write $w=b_{T}^{2} r$ for some $r \in \mathcal{P}_{k-5}(T)$. Finally using (4.3c), we obtain

$$
\int_{T} \operatorname{curl}\left(b_{T}^{2} r\right) \cdot \operatorname{curl}\left(b_{T}^{2} r\right) d x=\int_{T} \boldsymbol{v} \cdot \operatorname{curl}\left(b_{T}^{2} r\right) d x=0 .
$$

Clearly this last identity implies $r \equiv 0$ and therefore $\boldsymbol{v} \equiv 0$.

The global velocity and pressure space without boundary conditions are given respectively by

$$
\begin{aligned}
\boldsymbol{W}_{h} & =\left\{\boldsymbol{v} \in \boldsymbol{H}^{1}(\operatorname{div} ; \Omega): \nabla \boldsymbol{v} \text { is continuous at the vertices, and }\left.\boldsymbol{v}\right|_{T} \in \boldsymbol{W}(T) \forall T \in \mathcal{T}_{h}\right\}, \\
S_{h} & =\left\{q \in H^{1}(\Omega):\left.q\right|_{T} \in S(T) \forall T \in \mathcal{T}_{h}\right\} .
\end{aligned}
$$

As before, a counting argument can be used to show exactness of the second sequence given in (1.5). Recall that $\operatorname{dim} \Sigma_{h}=6 V+(2 k-7) E+\frac{1}{2}(k-3)(k-4) T$. Since $\operatorname{dim} S_{h}=V+(k-4) E+\frac{1}{2}(k-2)(k-3) T$ and $\operatorname{dim} \boldsymbol{W}_{h}=2(3 V+(k-3) E)+(k-4) E+\left(\frac{1}{2}(k-2)(k-3)-1\right) T+\frac{1}{2}(k-3)(k-4) T$, we easily find

$$
\begin{aligned}
& \operatorname{dim} \Sigma_{h}+\operatorname{dim} S_{h}- \operatorname{dim} \boldsymbol{V}_{h} \\
&=6 V+(2 k-7) E+\frac{1}{2}(k-3)(k-4) T+V+(k-4) E+\frac{1}{2}(k-2)(k-3) T \\
&-2(3 V+(k-3) E)-(k-4) E-\left(\frac{1}{2}(k-2)(k-3)-1\right) T \\
& \quad-\frac{1}{2}(k-3)(k-4) T=V-E+T=1 .
\end{aligned}
$$

We can then argue that the sequence (1.5) is exact.

Lemma 4.3. For any $q \in S_{h}$, there exists a $\boldsymbol{v} \in \boldsymbol{W}_{h}$ such that $\operatorname{div} \boldsymbol{v}=q$ and $\|\boldsymbol{v}\|_{H^{1}(\Omega)} \leq$ $C\|q\|_{L^{2}(\Omega)}$.

Proof. Since $q \in S_{h} \subset Q_{h}$, Lemma 3.1 states that there exists $\boldsymbol{v} \in \boldsymbol{V}_{h}$ such that $\operatorname{div} \boldsymbol{v}=q$ and $\|\boldsymbol{v}\|_{H^{1}(\Omega)} \leq C\|q\|_{L^{2}(\Omega)}$. By definition of $\boldsymbol{V}_{h}$, the function $\boldsymbol{v}$ is $C^{1}$ at the vertices of the triangulation. Therefore since $\operatorname{div} \boldsymbol{v}=q \in S_{h}$, we have $\boldsymbol{v} \in \boldsymbol{W}_{h}$. $\square$ 
4.2. The finite element spaces with homogeneous boundary conditions. The corresponding finite element spaces incorporating homogeneous boundary conditions are given by

$$
\begin{aligned}
\boldsymbol{W}_{h, 0} & =\left\{\boldsymbol{v} \in \boldsymbol{H}_{0}^{1}(\Omega):\left.\boldsymbol{v}\right|_{T} \in \boldsymbol{W}(T), \nabla \boldsymbol{v} \text { is continuous at all non-corner vertices }\right\}, \\
S_{h, 0} & =\left\{q \in \stackrel{\circ}{L}^{2}(\Omega):\left.q\right|_{T} \in S(T), q \text { is continuous at all non-corner vertices }\right\} .
\end{aligned}
$$

Lemma 4.4. Suppose that the mesh restriction (M) holds, and let $\boldsymbol{W}_{h, 0}$ and $S_{h, 0}$ be defined by (4.4a) and (4.4b) respectively. Then for any $q \in S_{h, 0}$ there exists $\boldsymbol{v} \in \boldsymbol{W}_{h, 0}$ with $\operatorname{div} \boldsymbol{v}=q$ and $\|\boldsymbol{v}\|_{H^{1}(\Omega)} \leq C\|q\|_{L^{2}(\Omega)}$.

The proof of Lemma 4.4 is nearly identical to the proof of Lemma 4.3 so we omit it.

5. The Finite Element Method and its Convergence Analysis. Let $\boldsymbol{X}_{h, 0} \times Y_{h, 0}$ be one of the velocity-pressure finite element pairs $\boldsymbol{V}_{h, 0} \times Q_{h, 0}$ or $\boldsymbol{W}_{h, 0} \times S_{h, 0}$. Then the finite element method reads: Find $\left(\boldsymbol{u}_{h}, p_{h}\right) \in \boldsymbol{X}_{h, 0} \times Y_{h, 0}$ such that

$$
\begin{aligned}
\nu \int_{\Omega} \nabla \boldsymbol{u}_{h}: \nabla \boldsymbol{v} d x- & \int_{\Omega}(\operatorname{div} \boldsymbol{v}) p_{h} d x=\int_{\Omega} \boldsymbol{f} \cdot \boldsymbol{v} d x & & \forall \boldsymbol{v} \in \boldsymbol{X}_{h, 0}, \\
\int_{\Omega}\left(\operatorname{div} \boldsymbol{u}_{h}\right) q d x & =0 & & \forall q \in Y_{h, 0} .
\end{aligned}
$$

In light of Lemmas 3.3 and 4.4, the LBB condition (1.2) holds provided the mesh condition (M) is satisfied. Using standard arguments [8,5], we conclude that problem (5.1) is well-posed. Moreover, since $\operatorname{div} \boldsymbol{X}_{h}=Y_{h}$ the space of discretely divergence-free functions is in fact divergence-free pointwise; that is,

$$
\boldsymbol{Z}_{h, 0}:=\left\{\boldsymbol{v} \in \boldsymbol{X}_{h, 0}: \int_{\Omega}(\operatorname{div} \boldsymbol{v}) q d x=0 \forall q \in Y_{h, 0}\right\}=\left\{\boldsymbol{v} \in \boldsymbol{X}_{h, 0}: \operatorname{div} \boldsymbol{v} \equiv 0\right\} .
$$

Therefore by standard arguments $[8,5]$ the solution satisfies the following estimates.

Lemma 5.1. Suppose that the mesh restriction (M) holds, and let $\left(\boldsymbol{u}_{h}, p_{h}\right) \in \boldsymbol{X}_{h, 0} \times Y_{h, 0}$ satisfy (5.1). Then there holds

$$
\begin{aligned}
\left\|\nabla\left(\boldsymbol{u}_{h}-\boldsymbol{v}\right)\right\|_{L^{2}(\Omega)} & \leq\|\nabla(\boldsymbol{u}-\boldsymbol{v})\|_{L^{2}(\Omega)} \quad \forall \boldsymbol{v} \in \boldsymbol{Z}_{h, 0}, \\
\left\|p_{h}-P_{h} p\right\|_{L^{2}(\Omega)} & \leq \nu\left\|\nabla\left(\boldsymbol{u}-\boldsymbol{u}_{h}\right)\right\|_{L^{2}(\Omega)}
\end{aligned}
$$

where $P_{h}: L^{2}(\Omega) \rightarrow Y_{h, 0}$ denotes the $L^{2}$ projection onto $Y_{h, 0}$, and $\boldsymbol{Z}_{h, 0}$ is defined by (5.2). Consequently, by the triangle and Poincare inequalities, we have

$$
\begin{aligned}
\left\|\boldsymbol{u}-\boldsymbol{u}_{h}\right\|_{H^{1}(\Omega)} & \leq C \inf _{\boldsymbol{v} \in \boldsymbol{Z}_{h, 0}}\|\boldsymbol{u}-\boldsymbol{v}\|_{H^{1}(\Omega)}, \\
\left\|p-p_{h}\right\|_{L^{2}(\Omega)} & \leq C\left(\left\|p-P_{h} p\right\|_{L^{2}(\Omega)}+\nu\left\|\nabla\left(\boldsymbol{u}-\boldsymbol{u}_{h}\right)\right\|_{L^{2}(\Omega)}\right) .
\end{aligned}
$$

We now address the approximation properties of $\boldsymbol{Z}_{h, 0}$. Since the sequences (1.5) are exact, we have $\boldsymbol{Z}_{h, 0}=\operatorname{curl} \Sigma_{h, 0}$. Moreover, since $\boldsymbol{u}$ is divergence-free, we may write $\boldsymbol{u}=\operatorname{curl} w$ for some $w \in H_{0}^{2}(\Omega)$. It then follows that

$$
\inf _{\boldsymbol{v} \in \boldsymbol{Z}_{h, 0}}\|\boldsymbol{u}-\boldsymbol{v}\|_{H^{1}(\Omega)}=\inf _{z \in \Sigma_{h, 0}}\|\operatorname{curl} w-\operatorname{curl} z\|_{H^{1}(\Omega)} \leq \inf _{z \in \Sigma_{h, 0}}\|w-z\|_{H^{2}(\Omega)} .
$$


Using this identity along with the approximation properties of $\Sigma_{h, 0}$ and the $L^{2}$ projection $P_{h}$, we obtain our main result.

TheOREM 5.2. Let $(\boldsymbol{u}, p)$ be the solution to the Stokes problem (1.1), and assume that $\boldsymbol{u} \in$ $\boldsymbol{H}^{s}(\Omega)$ with $1 \leq s \leq k+1$. Suppose that the mesh restriction $(\mathrm{M})$ is satisfied, and let $\left(\boldsymbol{u}_{h}, p_{h}\right) \in$ $\boldsymbol{X}_{h, 0} \times Y_{h, 0}$ be the solution to (5.1). Then there holds

$$
\left\|\boldsymbol{u}-\boldsymbol{u}_{h}\right\|_{H^{1}(\Omega)} \leq C h^{s-1}\|\boldsymbol{u}\|_{H^{s}(\Omega)} .
$$

If $\boldsymbol{X}_{h, 0} \times Y_{h, 0}=\boldsymbol{V}_{h, 0} \times Q_{h, 0}$, then the pressure error satisfies

$$
\left\|p-p_{h}\right\|_{L^{2}(\Omega)} \leq C\left(\nu h^{s-1}\|\boldsymbol{u}\|_{H^{s}(\Omega)}+h^{m}\|p\|_{H^{m}(\Omega)}\right) \quad 1 \leq s \leq k+1,0 \leq m \leq k .
$$

If $\boldsymbol{X}_{h, 0} \times Y_{h, 0}=\boldsymbol{W}_{h, 0} \times S_{h, 0}$, then the pressure error satisfies

$$
\left\|p-p_{h}\right\|_{L^{2}(\Omega)} \leq C\left(\nu h^{s-1}\|\boldsymbol{u}\|_{H^{s}(\Omega)}+h^{m}\|p\|_{H^{m}(\Omega)}\right) \quad 1 \leq s \leq k+1,0 \leq m \leq k-2 .
$$

5.1. A post-processed pressure solution. Theorem 5.2 indicates that the finite element pair $\boldsymbol{W}_{h, 0} \times S_{h, 0}$ approximates the velocity and pressure in an umbalanced way; namely, the order of convergence of the pressure is two less than the velocity. Here, we describe a simple local post-possessing procedure to recover $\mathcal{O}\left(h^{k}\right)$ accuracy. The price we pay is that the post-processed solution is no longer globally continuous, although we do not consider this a disadvantage from a practical viewpoint.

Let $S^{*}(T) \subset H^{1}(T)$ be an auxiliary finite dimensional space consisting of piecewise polynomials. Let $\left(\boldsymbol{u}_{h}, p_{h}\right)$ be the unique solution to (5.1) with $\boldsymbol{X}_{h, 0} \times Y_{h, 0}=\boldsymbol{W}_{h, 0} \times S_{h, 0}$. Then for a given $T \in \mathcal{T}_{h}$, we define $p_{h}^{*} \in S^{*}(T)$ as the unique function satisfying the local Neumman problem

$$
\begin{aligned}
\int_{T} \nabla p_{h}^{*} \cdot \nabla q d x & =\nu \int_{T}\left(\Delta \boldsymbol{u}_{h}\right) \cdot \nabla q d x+\int_{T} \boldsymbol{f} \cdot \nabla q d x \quad \forall q \in S^{*}(T), \\
\int_{T} p_{h}^{*} d x & =\int_{T} p_{h} d x .
\end{aligned}
$$

Lemma 5.3. Suppose that $\mathcal{P}_{k-1}(T) \subset S^{*}(T)$ and the solution to (1.1) satisfies $p \in H^{s-1}(\Omega)$ and $\boldsymbol{u} \in \boldsymbol{H}^{s}(\Omega)$ with $2 \leq s \leq k+1$. Then there holds

$$
\left\|p-p_{h}^{*}\right\|_{L^{2}(\Omega)} \leq C h^{s-1}\left(\nu\|\boldsymbol{u}\|_{H^{s}(\Omega)}+\|p\|_{H^{s-1}(\Omega)}\right) .
$$

Proof. By the Poincare-Friedrich's inequality, we have for each $T \in \mathcal{T}_{h}$,

$$
\left\|p-p_{h}^{*}\right\|_{L^{2}(T)} \leq C\left(\left\|\overline{p-p_{h}^{*}}\right\|_{L^{2}(T)}+h_{T}\left\|\nabla\left(p-p_{h}^{*}\right)\right\|_{L^{2}(T)}\right),
$$

where $\overline{p-p_{h}^{*}}$ denotes the mean of $p-p_{h}^{*}$ over $T$. Since $\overline{p-p_{h}^{*}}=\bar{p}-\bar{p}_{h}^{*}=\overline{P_{h} p}-\overline{p_{h}}=\overline{P_{h} p-p_{h}}$, we have $\left\|\overline{p-p_{h}^{*}}\right\|_{L^{2}(T)}=\left\|\overline{P_{h} p-p_{h}}\right\|_{L^{2}(T)} \leq\left\|P_{h} p-p_{h}\right\|_{L^{2}(T)}$.

To estimate $\left\|\nabla\left(p-p_{h}^{*}\right)\right\|_{L^{2}(T)}$, we use (5.5a) to get for all $q \in S^{*}(T)$

$$
\begin{aligned}
\left\|\nabla\left(p-p_{h}^{*}\right)\right\|_{L^{2}(T)}^{2}= & \left(\nabla\left(p-p_{h}^{*}\right), \nabla(p-q)\right)_{T}+\left(\nabla\left(p-p_{h}^{*}\right), \nabla\left(q-p_{h}^{*}\right)\right)_{T} \\
= & \left(\nabla\left(p-p_{h}^{*}\right), \nabla(p-q)\right)_{T}+\nu\left(\Delta\left(\boldsymbol{u}-\boldsymbol{u}_{h}\right), \nabla\left(q-p_{h}^{*}\right)\right)_{T} \\
\leq & \left\|\nabla\left(p-p_{h}^{*}\right)\right\|_{L^{2}(T)}\|\nabla(p-q)\|_{L^{2}(T)} \\
& \quad+\nu\left\|\Delta\left(\boldsymbol{u}-\boldsymbol{u}_{h}\right)\right\|_{L^{2}(T)}\left\|\nabla\left(q-p_{h}^{*}\right)\right\|_{L^{2}(T)} .
\end{aligned}
$$


It then follows from the triangle and Cauchy inequalities that

$$
\left\|\nabla\left(p-p_{h}^{*}\right)\right\|_{L^{2}(T)} \leq C\left(\|\nabla(p-q)\|_{L^{2}(T)}+\nu\left\|\Delta\left(\boldsymbol{u}-\boldsymbol{u}_{h}\right)\right\|_{L^{2}(T)}\right) .
$$

Using this last estimate in (5.6) we obtain

$$
\begin{gathered}
\left\|p-p_{h}^{*}\right\|_{L^{2}(T)} \leq C\left(\left\|p_{h}-P_{h} p\right\|_{L^{2}(T)}+\nu h_{T}\left\|\Delta\left(\boldsymbol{u}-\boldsymbol{u}_{h}\right)\right\|_{L^{2}(T)}\right. \\
\left.+\inf _{q \in S^{*}(T)} h_{T}\|\nabla(p-q)\|_{L^{2}(T)}\right) .
\end{gathered}
$$

If $\mathcal{P}_{k-1}(T) \subset S^{*}(T)$ then by the Bramble-Hilbert Lemma and the regularity properties of $p$,

$$
\inf _{q \in S^{*}(T)} h_{T}\|\nabla(p-q)\|_{L^{2}(T)} \leq C h_{T}^{s-1}\|p\|_{H^{s-1}(T)}, \quad 1 \leq s \leq k+1 .
$$

Moreover, by the triangle and inverse inequalities, we have for any $\boldsymbol{v} \in \mathcal{P}_{k}(T)$,

$$
\begin{aligned}
h_{T}\left\|\Delta\left(\boldsymbol{u}-\boldsymbol{u}_{h}\right)\right\|_{L^{2}(T)} & \leq h_{T}\|\Delta(\boldsymbol{u}-\boldsymbol{v})\|_{L^{2}(T)}+C\left\|\boldsymbol{u}_{h}-\boldsymbol{v}\right\|_{H^{1}(T)} \\
& \leq C\left(h_{T}\|\Delta(\boldsymbol{u}-\boldsymbol{v})\|_{L^{2}(T)}+\|\boldsymbol{u}-\boldsymbol{v}\|_{H^{1}(T)}+\left\|\boldsymbol{u}-\boldsymbol{u}_{h}\right\|_{H^{1}(T)}\right) .
\end{aligned}
$$

Thus, by choosing $\boldsymbol{v}$ to be the Scott-Zhang interpolant of $\boldsymbol{u}$ we obtain

$$
h_{T}\left\|\Delta\left(\boldsymbol{u}-\boldsymbol{u}_{h}\right)\right\|_{L^{2}(T)} \leq C\left(h_{T}^{s-1}\|\boldsymbol{u}\|_{H^{s}(\omega(T))}+\left\|\boldsymbol{u}-\boldsymbol{u}_{h}\right\|_{H^{1}(T)}\right) .
$$

Combining these results, we have

$$
\left\|p-p_{h}^{*}\right\|_{L^{2}(T)} \leq C\left(\left\|p_{h}-P_{h} p\right\|_{L^{2}(T)}+\nu\left\|\boldsymbol{u}-\boldsymbol{u}_{h}\right\|_{H^{1}(T)}+h_{T}^{s-1}\left(\nu\|\boldsymbol{u}\|_{H^{s}(T)}+\|p\|_{H^{s-1}(T)}\right)\right) .
$$

Summing over all $T \in \mathcal{T}_{h}$ and employing the estimates (5.3b) and (5.4), we obtain the desired result:

$$
\begin{aligned}
\left\|p-p_{h}^{*}\right\|_{L^{2}(\Omega)} & \leq C\left(\left\|p_{h}-P_{h} p\right\|_{L^{2}(\Omega)}+\nu\left\|\boldsymbol{u}-\boldsymbol{u}_{h}\right\|_{H^{1}(\Omega)}+h^{s-1}\left(\nu\|\boldsymbol{u}\|_{H^{s}(\Omega)}+\|p\|_{H^{s-1}(\Omega)}\right)\right) \\
& \leq C\left(\nu\left\|\boldsymbol{u}-\boldsymbol{u}_{h}\right\|_{H^{1}(\Omega)}+h^{s-1}\left(\nu\|\boldsymbol{u}\|_{H^{s}(\Omega)}+\|p\|_{H^{s-1}(\Omega)}\right)\right) \\
& \leq C h^{s-1}\left(\nu\|\boldsymbol{u}\|_{H^{s}(\Omega)}+\|p\|_{H^{s-1}(\Omega)}\right) .
\end{aligned}
$$

$\square$

6. Reduced Elements. In this section we briefly describe how to modify the finite element spaces in the case $k=4$ to obtain elements with less degrees of freedom. Essentially this is done by restricting the range of the tangental component on each edge. These modifications correspond to the $C^{1}$ Bell triangle [12] in the sequence (1.5).

The local spaces of the reduced velocity elements are given by

$$
\begin{aligned}
\boldsymbol{V}_{R}(T) & =\left\{\boldsymbol{v} \in \mathcal{P}_{4}(T):\left.\boldsymbol{v} \cdot \boldsymbol{t}\right|_{\partial T} \in \mathcal{P}_{3}(\partial T)\right\}, \\
\boldsymbol{W}_{R}(T) & =\boldsymbol{W}(T) \cap \boldsymbol{V}_{R}(T),
\end{aligned}
$$

where $\boldsymbol{t}$ denotes the unit tangent vector of $\partial T$, and it is understood that $k=4$ in the definition of $\boldsymbol{W}(T)$. The corresponding pressure spaces are the same as the unreduced elements with $k=4$. 
The 21 degrees of freedom for a function $\boldsymbol{v} \in \boldsymbol{W}_{R}(T)$ are (i) the values of $\boldsymbol{v}$ and $\nabla \boldsymbol{v}$ at the vertices; (ii) the zeroth moment of $\boldsymbol{v} \cdot \boldsymbol{n}$ on each edge. In addition to these DOFs, the degrees of freedom of $\boldsymbol{v} \in \boldsymbol{V}_{R}(T)$ are (iii) the zero and first order moments of $\boldsymbol{v}$ on $T$. The proofs of unisolvency are essentially the same as the proof of Lemma 4.2 so we omit them.

The corresponding global spaces, which consists of functions that are continuous at the degrees of freedom listed above, are denoted by $\boldsymbol{V}_{R, h}$ and $\boldsymbol{W}_{R, h}$. The proofs of stability for the finite element pairs $\boldsymbol{V}_{R, h} \times Q_{h}$ and $\boldsymbol{W}_{R, h} \times S_{h}$ are essentially the same as the proofs of Lemmas 3.1 and 4.3, the key difference being that equation (3.4) is replaced by

$$
\int_{e} \boldsymbol{v}_{1} \cdot \boldsymbol{n}_{e} d s=\int_{e} \boldsymbol{w} \cdot \boldsymbol{n}_{e} d s .
$$

The convergence analysis also follows the same lines as those presented in Section 5. Following the arguments there, we find that the velocity approximations converge with order $\mathcal{O}\left(h^{3}\right)$; the pressure approximations converge with order $\mathcal{O}\left(h^{3}\right)$ or $\mathcal{O}\left(h^{2}\right)$ depending on what finite element pair is used.

It is worth comparing the size of the global reduced finite element space $\boldsymbol{W}_{R, h} \times S_{h}$ with some well-known Stokes elements. The global dimension of $\boldsymbol{W}_{R, h} \times S_{h}$ is $\operatorname{dim} \boldsymbol{W}_{R, h}+\operatorname{dim} S_{h}=$ $(6 V+E)+(V+T)=7 V+T+E=8 V+2 T-1$. A comparable element in terms of approximation properties is the $\mathcal{P}_{3}-\mathcal{P}_{2}$ Taylor hood finite element space (where both spaces are continuous). The dimension of this space is $2(V+2 E+T)+V+E=3 V+5 E+2 T=8 V+7 T-1$, so we clearly see that $\boldsymbol{W}_{R, h} \times S_{h}$ has smaller dimension, although the pressure approximation converges with one less order than the Taylor-Hood approximation without post processing. However, we recall that the Taylor-Hood elements do not produce exactly divergence-free approximations. Another comparable element is the Scott-Vogelius element on a mesh created as a barycenter refinement of a triangular mesh. For such meshes, it has been shown that the $\mathcal{P}_{2}-\mathcal{P}_{1}$ pair with $\mathcal{P}_{2}$ continuous and $\mathcal{P}_{1}$ discontinuous satisfy the LBB condition (1.2) and produce exactly divergence-free velocity approximations. The dimension of this space is $2 V+2 E+3 T=4 V+5 T-2$ which is $\approx 16.7 \%$ larger than the dimension of $\boldsymbol{W}_{R, h} \times S_{h}$. In addition, $\boldsymbol{W}_{R, h} \times S_{h}$ has better approximation properties.

7. Numerical Experiments. In this section we perform some numerical experiments which back up the theoretical results in Section 5. We apply the finite element method (5.1) with $k=4$ on the unit square $\Omega=(0,1)^{2}$. The computations are performed on a criss-cross triangulation as depicted in Figure 7.1. These meshes have approximately $h^{-2}$ singular vertices, but none of these singular vertices are located at the corners of the domain. Hence these triangulations satisfy the mesh restriction $(\mathrm{M})$.

We choose the data such that the exact solution is given by

$$
\begin{aligned}
& \boldsymbol{u}=\exp \left(x_{1}^{4}-x_{2}^{3}\right)\left(\begin{array}{c}
3\left(1-\cos ^{2}\left(3 \pi x_{1}\right)\right) \sin \left(3 \pi x_{2}\right)\left(2 \pi \cos \left(3 \pi x_{2}\right)-x_{2}^{2} \sin \left(3 \pi x_{2}\right)\right) \\
2\left(-1+\cos ^{2}\left(3 \pi x_{2}\right)\right) \sin \left(3 \pi x_{1}\right)\left(3 \pi \cos \left(3 \pi x_{1}\right)+2 x_{1}^{3} \sin \left(3 \pi x_{1}\right)\right)
\end{array}\right), \\
& p=10\left(x_{1}^{5}-x_{2}^{3}+\frac{1}{12}\right) .
\end{aligned}
$$

The viscosity is set as $\nu=1$.

In the first set of numerical experiments, we take $\boldsymbol{X}_{h, 0} \times Y_{h, 0}=\boldsymbol{V}_{h, 0} \times Q_{h, 0}$. We list the errors and the rates of convergence of the computed solution in Table 7.1. In addition, we also list the maximum value of the divergence of the computed velocity solution. Table 7.1 clearly indicates that the $\left\|\boldsymbol{u}-\boldsymbol{u}_{h}\right\|_{H^{1}(\Omega)}=\mathcal{O}\left(h^{4}\right)$ and $\left\|p-p_{h}\right\|_{L^{2}(\Omega)}=\mathcal{O}\left(h^{4}\right)$ which is in agreement with Theorem 5.2. In addition, we observe that the velocity error satisfies $\left\|\boldsymbol{u}-\boldsymbol{u}_{h}\right\|_{L^{2}(\Omega)}=\mathcal{O}\left(h^{5}\right)$. Finally, we 
see from Table 7.1 that the divergence of the computed solution is of the order $10^{-9}-10^{-12}$ and increases as the mesh is refined. This phenomenon is likely due to round-off error.

Table 7.2 shows the resulting errors when we compute the solution of (5.1) with finite element spaces $\boldsymbol{X}_{h, 0} \times Y_{h, 0}=\boldsymbol{W}_{h, 0} \times S_{h, 0}$. Again, we observe $\mathcal{O}\left(h^{5}\right)$ and $\mathcal{O}\left(h^{4}\right)$ rates of convergence of the velocity error in the $L^{2}$ and $H^{1}$ norms, respectively. The pressure converges quadratically with respect to $h$. These rates coincide with the statements in Theorem 5.2.

Finally, in Table 7.3 we list the errors of the post-processed solution $p_{h}^{*}$ with $S(T)=\mathcal{P}_{3}(T)$. In addition, we also list the quantity $\left\|p_{h}-P_{h} p\right\|_{L^{2}(\Omega)}$. As predicted by Lemmas 5.1 and 5.3 , both of these quantities converge with order $\mathcal{O}\left(h^{4}\right)$.

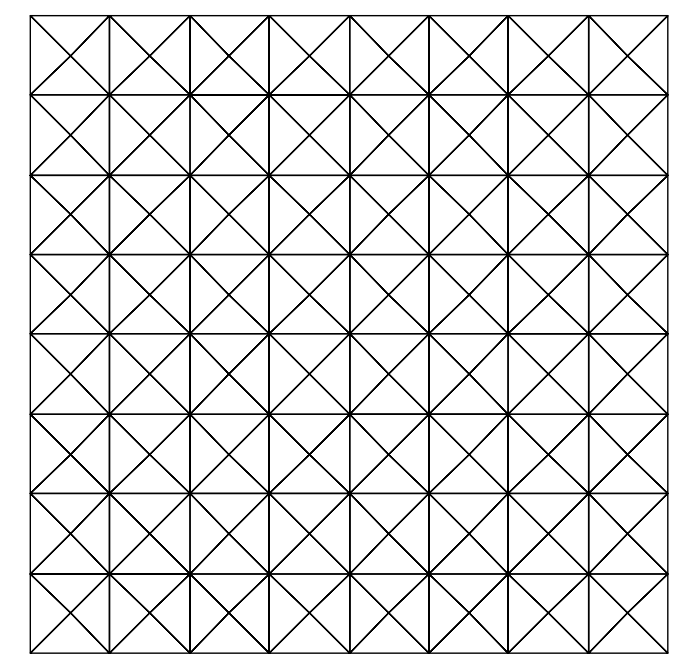

FIG. 7.1. The computational mesh with $h=1 / 8$.

TABLE 7.1

The errors of the computed solution and rates of convergence with finite element spaces $\boldsymbol{V}_{h, 0} \times Q_{h, 0}$.

\begin{tabular}{cccccccc}
$h$ & $\left\|\boldsymbol{u}-\boldsymbol{u}_{h}\right\|_{L^{2}}$ & rate & $\left\|\nabla\left(\boldsymbol{u}-\boldsymbol{u}_{h}\right)\right\|_{L^{2}}$ & rate & $\left\|p-p_{h}\right\|_{L^{2}}$ & rate & $\left\|\operatorname{div} \boldsymbol{u}_{h}\right\|_{L^{\infty}}$ \\
\hline $1 / 4$ & $2.16 \mathrm{E}-01$ & & $1.18 \mathrm{E}+01$ & & $1.24 \mathrm{E}+02$ & & $1.28 \mathrm{E}-11$ \\
$1 / 8$ & $1.95 \mathrm{E}-02$ & 3.47 & $1.68 \mathrm{E}+00$ & 2.81 & $1.20 \mathrm{E}+01$ & 3.36 & $6.27 \mathrm{E}-12$ \\
$1 / 16$ & $6.38 \mathrm{E}-04$ & 4.93 & $1.18 \mathrm{E}-01$ & 3.83 & $1.90 \mathrm{E}+00$ & 2.66 & $8.92 \mathrm{E}-11$ \\
$1 / 32$ & $2.03 \mathrm{E}-05$ & 4.97 & $7.82 \mathrm{E}-03$ & 3.91 & $1.36 \mathrm{E}-01$ & 3.81 & $1.45 \mathrm{E}-11$ \\
$1 / 64$ & $6.58 \mathrm{E}-07$ & 4.95 & $5.13 \mathrm{E}-04$ & 3.93 & $8.76 \mathrm{E}-03$ & 3.96 & $9.83 \mathrm{E}-10$
\end{tabular}

8. Conclusions. In this paper we developed two families of finite element pairs for the Stokes problem that produce exactly divergence-free velocity approximations. The construction of both families are both guided by two different Stokes complexes, where a $C^{1}$ family of finite elements plays a central role. In three dimensions, we expect that a smooth de Rham complex (i.e., Stokes complex) will also play an integral role in both the construction and analysis of divergence-free Stokes elements. We plan to address the three-dimensional case in the near future.

We end the paper discussing how by using the techniques of Lemmas 3.1 and 4.3 , it can be 
TABLE 7.2

The errors of the computed solution and rates of convergence with finite element spaces $\boldsymbol{W}_{h, 0} \times S_{h, 0}$.

\begin{tabular}{cccccccc}
$h$ & $\left\|\boldsymbol{u}-\boldsymbol{u}_{h}\right\|_{L^{2}}$ & rate & $\left\|\nabla\left(\boldsymbol{u}-\boldsymbol{u}_{h}\right)\right\|_{L^{2}}$ & rate & $\left\|p-p_{h}\right\|_{L^{2}}$ & rate & $\|$ div $\boldsymbol{u}_{h} \|_{L^{\infty}}$ \\
\hline $1 / 4$ & $2.16 \mathrm{E}-01$ & & $1.18 \mathrm{E}+01$ & & $1.60 \mathrm{E}+00$ & & $3.65 \mathrm{E}-11$ \\
$1 / 8$ & $1.95 \mathrm{E}-02$ & 3.47 & $1.68 \mathrm{E}+00$ & 2.81 & $4.38 \mathrm{E}-01$ & 1.86 & $1.84 \mathrm{E}-11$ \\
$1 / 16$ & $6.38 \mathrm{E}-04$ & 4.93 & $1.18 \mathrm{E}-01$ & 3.83 & $2.00 \mathrm{E}-01$ & 1.13 & $1.12 \mathrm{E}-11$ \\
$1 / 32$ & $2.03 \mathrm{E}-05$ & 4.97 & $7.82 \mathrm{E}-03$ & 3.91 & $5.66 \mathrm{E}-02$ & 1.82 & $1.24 \mathrm{E}-11$ \\
$1 / 64$ & $6.58 \mathrm{E}-07$ & 4.95 & $5.13 \mathrm{E}-04$ & 3.93 & $1.48 \mathrm{E}-02$ & 1.94 & $5.79 \mathrm{E}-11$
\end{tabular}

TABLE 7.3

The difference between the computed pressure and the $L^{2}$ projection of $p$, and the errors of the post-processed pressure solution with $S(T)=\mathcal{P}_{3}(T)$.

\begin{tabular}{ccccc}
$h$ & $\left\|p_{h}-P_{h} p\right\|_{L^{2}}$ & rate & $\left\|p-p_{h}^{*}\right\|_{L^{2}(\Omega)}$ & rate \\
\hline $1 / 4$ & $1.19 \mathrm{E}+00$ & & $1.80 \mathrm{E}+01$ & \\
$1 / 8$ & $1.27 \mathrm{E}-01$ & 3.23 & $2.44 \mathrm{E}+00$ & 2.88 \\
$1 / 16$ & $1.05 \mathrm{E}-02$ & 3.60 & $1.88 \mathrm{E}-01$ & 3.70 \\
$1 / 32$ & $5.85 \mathrm{E}-04$ & 4.16 & $1.30 \mathrm{E}-02$ & 3.86 \\
$1 / 64$ & $3.26 \mathrm{E}-05$ & 4.17 & $9.19 \mathrm{E}-04$ & 3.82
\end{tabular}

shown that the Scott-Vogelius elements are uniformly LBB stable without the quasi-uniform mesh assumption. Furthermore, the proof is shorter and simpler than the one given in [22, 25]. We sketch the main points in the argument.

Let $q \in L^{2}(\Omega)$ be a given piecewise polynomial of degree $k-1$ with $k \geq 4$. We wish to construct a globally continuous piecewise polynomial $\boldsymbol{v} \in \mathcal{P}_{k}$ such that $\operatorname{div} \boldsymbol{v}=q$ and $\|\boldsymbol{v}\|_{H^{1}(\Omega)} \leq C\|q\|_{L^{2}(\Omega)}$. Let $\boldsymbol{w} \in \boldsymbol{H}^{1}(\Omega)$ satisfy $\operatorname{div} \boldsymbol{w}=q$ and $\|\boldsymbol{w}\|_{H^{1}(\Omega)} \leq C\|q\|_{L^{2}(\Omega)}$. By slightly modifying the proof of [25, Lemma 2.6] (also see [22, Lemma 4.1]), we can easily construct a $\boldsymbol{v}_{1} \in \mathcal{P}_{k}$ such that $\operatorname{div} \boldsymbol{v}_{1}=q$ at the vertices (provided the mesh does not contain any singular vertices) and $\int_{e} \boldsymbol{v}_{1} \cdot \boldsymbol{\kappa} d s=$ $\int_{e} \boldsymbol{w} \cdot \boldsymbol{\kappa}$ for all $\boldsymbol{\kappa} \in \mathcal{P}_{k-4}(e)$ and $e \in \mathcal{E}_{h}$. Furthermore by scaling, we have $\left\|\boldsymbol{v}_{1}\right\|_{H^{1}(\Omega)} \leq C\|q\|_{L^{2}(\Omega)}$, where the constant $C>0$ depends on "the measure of singularity" of the triangulation (cf. [22]). Consequently, by the divergence theorem, $\int_{T} \operatorname{div} \boldsymbol{v}_{1} d x=\int_{T} q d x$. Employing Lemma 2.3, we find a $\boldsymbol{v}_{2, T} \in \boldsymbol{P}_{k}(T) \cap \boldsymbol{H}_{0}^{1}(T)$ with $\left.\operatorname{div} \boldsymbol{v}_{2, T}\right|_{T}=\left.\left(q-\operatorname{div} \boldsymbol{v}_{1}\right)\right|_{T}$. The function $\boldsymbol{v}=\boldsymbol{v}_{1}+\boldsymbol{v}_{2}$ with $\left.\boldsymbol{v}_{2}\right|_{T}=\left.\boldsymbol{v}_{2, T}\right|_{T}$ then satisfies the desired properties $\operatorname{div} \boldsymbol{v}=q$ and $\|\boldsymbol{v}\|_{H^{1}(\Omega)} \leq C\|q\|_{L^{2}(\Omega)}$.

\section{REFERENCES}

[1] J.H. Argyris, I. Fried, And D.W. Scharpf, The TUBA family of plate elements for the matrix displacement method, Aero. J. Roy. Aero. Soc., 72, 701-709, 1968.

[2] D.N. Arnold, F. Brezzi, And M. Fortin, A stable finite element for the Stokes equations, Calcolo, 21:337-344, 1984.

[3] D.N. Arnold and J. Qin, Quadratic velocity/linear pressure Stokes elements, in Advances in Computer Methods for Partial Differential Equations VII, ed. R. Vichnevetsky and R.S. Steplemen, 1992.

[4] C. Bernardi and G. Raugel, Analysis of some finite elements for the Stokes problem, Math. Comp., 44(169):71-79, 1985.

[5] D. Boffi, F. Brezzi And M. Fortin, Finite elements for the Stokes problem, in Mixed Finite Elements, Compatibility Conditions, and Applications, Lectures given at the C.I.M.E. Summer School, SpringerVerlag, Berlin, 2008.

[6] D. Boffi, N. Cavallini, F. Gardini, and L. Gastaldi, Local mass conservation of Stokes finite elements, J. 
Sci. Comput., 52(2):383-400, 2012

[7] S.C. Brenner And L.R. Scott, The Mathematical Theory of Finite Element Methods (Third edition), Springer, 2008.

[8] F. Brezzi And M. Fortin, Mixed and Hybrid Finite Element Methods, Springer Series in Computational Mathematics,15, Springer-Verlag, New York, 1991.

[9] J. Carrero, B. Cockburn, and D. Schötzau, Hybridized globally divergence-free LDG methods. I. The Stokes problem, Math. Comp., 75:533-563, 2006.

[10] B. Cockburn, G. Kanschat, And D. Schötzau, A note on discontinuous Galerkin divergence-free solutions of the Navier-Stokes equations, J. Sci. Comput., 31(1-2):61-73, 2007.

[11] M.A. Case, V.J. Ervin, A. Linke, L.G. Rebholz, A connection between Scott-Vogelius and grad-div stabilized Taylor-Hood FE approximations of the Navier-Stokes equations, SIAM J. Numer. Anal., 49(4):1461-1481, 2011.

[12] P. G. Ciarlet, The Finite Element Method for Elliptic Problems, North-Holland, Amsterdam, 1978.

[13] J.A. Evans, AND T.J.R. Hughes, Isogeometric divergence-conforming B-splines for the steady Navier-Stokes equations, Mathematical Models and Methods in Applied Sciences, DOI: 10.1142/S0218202513500139, 2012.

[14] J.A. Evans, And T.J.R. Hughes, Isogeometric divergence-conforming B-splines for the unsteady Navier-Stokes equations, Journal of Computational Physics, to appear.

[15] V. Girault and P.-A. Raviart, Finite element methods for the Navier-Stokes equations, Berlin, SpringerVerlag, 1986.

[16] J. Guzmán and M. Neilan, Conforming and divergence-free Stokes elements on general triangular meshes, Math. Comp. (to appear).

[17] A. Linke, Collision in a cross-shaped domain - A steady 2d Navier Stokes example demonstrating the importance of mass conservation in CFD, Comput. Methods Appl. Mech. Engrg., 198:3278-3286, 2009.

[18] A. Linke, Divergence-Free Mixed Finite Elements for the Incompressible Navier-Stokes Equation, Ph.D. Thesis, University of Erlangen, 2008.

[19] K.A. Mardal, X.-C. Tai and R. Winther, A robust finite element method for Darcy-Stokes flow, SIAM J. Numer. Anal. 40:1605-1631, 2002.

[20] K.A. Mardal, J. Schöberl, and R. Winther, A uniform inf-sup condition with applications to preconditioning, preprint, arXiv:1201.1513 [math.NA], arXiv, 2012.

[21] M.A. Olshanskit, And A. Reusken, Grad-div stabilization for Stokes equations, Math. Comp., 73(248):1699$1718,2004$.

[22] L.R. ScOtt And M. Vogelius, Norm estimates for a maximal right inverse of the divergence operator in spaces of piecewise polynomials, Math. Model. Numer. Anal., 9:11-43, 1985.

[23] L.R. Scott And S. Zhang, Finite element interpolation of non smooth functions satisfying boundary conditions, Math. Comp., 54(190):483-493, 1990.

[24] X.-C. TAi And R. Winther, A discrete de Rham complex with enhanced smoothness, Calcolo 43:287-306, 2006.

[25] M. Vogelius, A right-inverse for the divergence operator in spaces of piecewise polynomials. Application to the p-version of the finite element method, Numer. Math., 41:19-37, 1983 